\title{
Polymer-supported Mukaiyama reagent: a useful coupling reagent for the synthesis of esters and amides.
}

\author{
Stefano Crosignani, Jerome Gonzalez and Dominique Swinnen* \\ Serono PharmaceuticalResearch Institute, 14 Chemin des Aulx, 1228 Plan-les-Ouates, Geneva, Switzerland. \\ dominique.swinnen@serono.com
}

\section{Supporting information}

General: ${ }^{1} \mathrm{H}-\mathrm{NMR}$ spectra were recorded using a BRUKER DPX-300 spectrometer (300 MHz). HPLC analysis were performed on a Waters 2695 instrument, equipped with a Waters 996 Photodiode Array Detector using an XTerra MSC8 $3.5 \mu \mathrm{m} 4.6 \times 50 \mathrm{~mm}$ column. Chromatographic conditions: gradient from $95 \% \mathrm{H}_{2} \mathrm{O}\left(0.1 \%\right.$ TFA) : $5 \% \mathrm{CH}_{3} \mathrm{CN}\left(0.1 \%\right.$ TFA) to $5 \% \mathrm{H}_{2} \mathrm{O}\left(0.1 \%\right.$ TFA) : $95 \% \mathrm{CH}_{3} \mathrm{CN}(0.1 \%$ TFA) over 8 minutes, flow $2 \mathrm{~mL} / \mathrm{min}$. Mass spectra were determined on a Micromass ZMD (electrospray, positive or negative ionisation). All reagents were purchased and used without further purification. Microwave-assisted reactions were performed on a Smith Synthesizer ${ }^{\mathrm{TM}}$.

\section{Preparation of resin 1}

Wang resin $(5.00 \mathrm{~g}, 8.5 \mathrm{mmol})$ is suspended in dry DCM $(50 \mathrm{~mL})$. 2-Chloropyridine $(4.0 \mathrm{~mL}, 42.5$ mmol, $5 \mathrm{eq})$ is added and the mixture cooled with an ice-bath. Trifluoromethanesulfonic anhydride (2.0 $\mathrm{mL}, 11.9 \mathrm{mmol}, 1.4 \mathrm{eq}$ ) is added dropwise and after 5 minutes the ice-bath is remover. The mixture is stirred overnight at room temperature. The resin is collected by filtration and washed with DCM, DMF and DCM, and dried under vacuum.

\section{Synthesis of amides using resin 1}

The carboxylic acid $(0.16 \mathrm{mmol}, 1.05 \mathrm{eq})$, the amine $(0.15 \mathrm{mmol}, 1 \mathrm{eq})$ and TEA $(0.45 \mathrm{mmol}, 3 \mathrm{eq})$ are dissolved in anhydrous DCM (3 mL). Resin 1 (240 mg, $0.3 \mathrm{mmol}, 2 \mathrm{eq})$ is added and stirring is continued until completion of the reaction (judged by HPLC, TLC or NMR). The resin is removed by filtration, and the filtrates are passed through an amino-functionalized SPE column (Isolute ${ }^{\circledR} \mathrm{NH}_{2} \mathrm{SPE}$ Column, 2 g, Part No. 470-0200-D). The resin and SPE column are washed with $10 \mathrm{~mL}$ of DCM, then the combined filtrates are evaporated under vacuum to afford the desired amide. 


\section{Synthesis of esters using resin 1}

The carboxylic acid $(0.16 \mathrm{mmol}, 1.05 \mathrm{eq})$, the alcohol $(0.15 \mathrm{mmol}, 1 \mathrm{eq})$ and TEA $(0.45 \mathrm{mmol}, 3 \mathrm{eq})$ are dissolved in anhydrous DCM (3 mL). PS-DMAP (20 mg, $0.03 \mathrm{mmol}, 0.2 \mathrm{eq})$ is added, followed by resin 1 (240 mg, $0.3 \mathrm{mmol}, 2 \mathrm{eq}$ ) and stirring is continued until completion of the reaction (judged by HPLC, TLC or NMR). The resin is removed by filtration, and the filtrates are passed through an aminofunctionalized SPE column (Isolute ${ }^{\circledR} \mathrm{NH}_{2}$ SPE Column, 2 g, Part No. 470-0200-D). The resin and SPE column are washed with $10 \mathrm{~mL}$ of DCM, then the combined filtrates are evaporated under vacuum to afford the desired ester.

\section{Modified procedure for the synthesis of phenolic esters (Table 2, entries 14 and 15)}

The carboxylic acid $(0.16 \mathrm{mmol}, 1.05 \mathrm{eq})$, the alcohol $(0.15 \mathrm{mmol}, 1 \mathrm{eq})$ and TEA $(0.45 \mathrm{mmol}, 3 \mathrm{eq})$ are dissolved in anhydrous DCM (3 mL). PS-DMAP (20 mg, $0.03 \mathrm{mmol}, 0.2 \mathrm{eq})$ is added, followed by resin 1 (240 mg, $0.3 \mathrm{mmol}, 2 \mathrm{eq}$ ) and stirring is continued until completion of the reaction (judged by HPLC, TLC or NMR). The resin is removed by filtration and washed with $10 \mathrm{~mL}$ of DCM. The organic phase is extracted twice with sat. $\mathrm{NaHCO}_{3}$ and the aqueous phases are back-extracted with DCM. The combined organic phases are dried over $\mathrm{MgSO}_{4}$ and evaporated under vacuum to afford the desired phenolic ester. 


\section{Characterisation of all products}

\section{Table 1 entry 1}

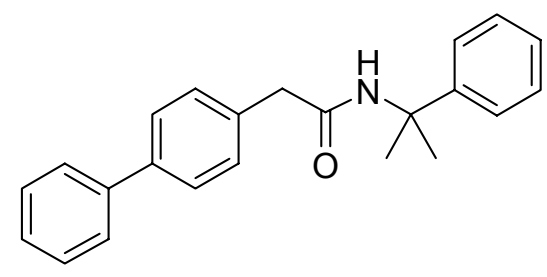

${ }^{1} \mathrm{H}$ NMR $\left(300 \mathrm{MHz} ; \mathrm{CDCl}_{3}\right) \delta_{\mathrm{H}}$ : 7.65-7.62 (4H, m); 7.51-7.46 (2H, m); 7.42-7.37 (3H, m); 7.34-7.32 $(4 \mathrm{H}, \mathrm{m}) ; 7.30-7.21(1 \mathrm{H}, \mathrm{m}), 5.82(1 \mathrm{H}, \mathrm{bs}) ; 3.60(2 \mathrm{H}, \mathrm{s}) ; 3.51(6 \mathrm{H}, \mathrm{s})$.

HPLC retention times $4.41 \mathrm{~min}$. ESMS m/z $330.26(\mathrm{M}+\mathrm{H})^{+}$

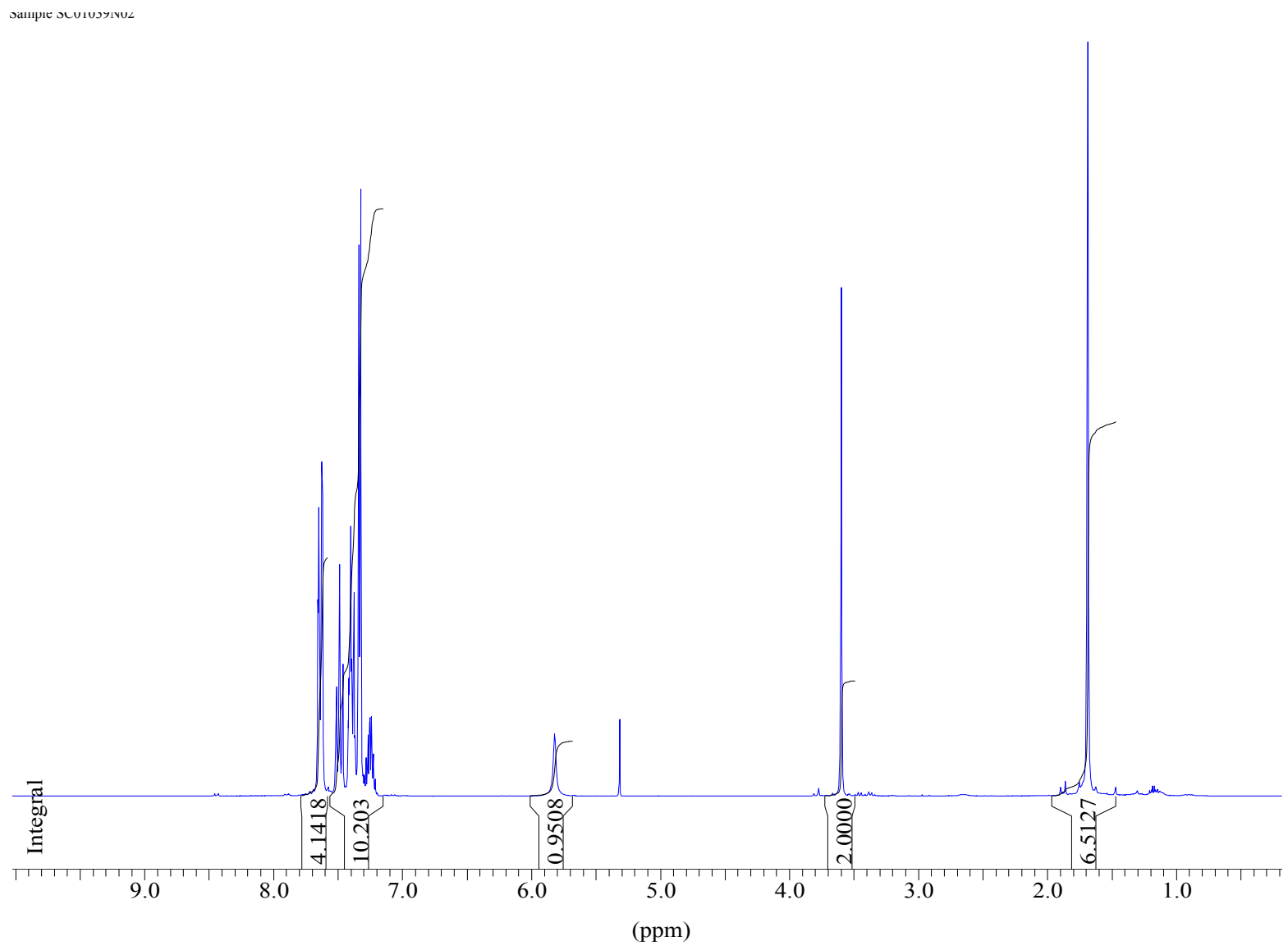




\section{Table 1 entry 2}<smiles>CC(=O)OC(C)(C)[NH2+][C@H](Cc1c[nH]c2ccccc12)C(=O)N[C@@H](C(C)=O)C(C)C</smiles>

${ }^{1} \mathrm{H}$ NMR $\left(300 \mathrm{MHz} ; \mathrm{CDCl}_{3}\right) \delta_{\mathrm{H}}: 8.12(1 \mathrm{H}, \mathrm{bs}) ; 7.58(2 \mathrm{H}, \mathrm{d}, J=7.5 \mathrm{~Hz}) ; 7.28(2 \mathrm{H}, \mathrm{d}, J=7.5 \mathrm{~Hz}) ; 7.15-$ $7.00(3 \mathrm{H}, \mathrm{m}) ; 6.22(1 \mathrm{H}, \mathrm{d}, J=8.5 \mathrm{~Hz}) ; 5.11(1 \mathrm{H}, \mathrm{bs}) ; 4.40-4.34(1 \mathrm{H}, \mathrm{m}) ; 4.33(1 \mathrm{H}, \mathrm{dd}, J=8.5 \mathrm{~Hz}, J=5.0$ $\mathrm{Hz}) ; 3.56(3 \mathrm{H}, \mathrm{s}) ; 3.22(1 \mathrm{H}, \mathrm{dd}, J=14.5 \mathrm{~Hz}, J=6.0 \mathrm{~Hz}) ; 3.12(1 \mathrm{H}, \mathrm{dd}, J=14.5 \mathrm{~Hz}, J=7.5 \mathrm{~Hz}) ; 1.95(1 \mathrm{H}$, $\mathrm{m}) ; 1.36(9 \mathrm{H}, \mathrm{s}) ; 0.72(3 \mathrm{H}, \mathrm{d}, J=7.0 \mathrm{~Hz}) ; 0.70(3 \mathrm{H}, \mathrm{d}, J=7.0 \mathrm{~Hz})$.

HPLC retention times $3.93 \mathrm{~min}$.

Diastereomeric excess measured by HPLC using an XTerra MSC8 $5 \mu \mathrm{m} 4.6 \times 250 \mathrm{~mm}$ column. Chromatographic conditions: isocratic elution $95 \% \mathrm{H}_{2} \mathrm{O}(0.1 \%$ TFA $): 5 \% \mathrm{CH}_{3} \mathrm{CN}(0.1 \% \mathrm{TFA})$. Retention times (min): 20.35 (minor) 21.29 (major)






\section{Table 1 entry 3}<smiles>CC(=O)N[C@@H](Cc1c[nH]c2ccccc12)C(=O)N[C@@H](C(=O)OC(C)=O)C(C)C</smiles>

${ }^{1} \mathrm{H}$ NMR $\left(300 \mathrm{MHz} ; \mathrm{CDCl}_{3}\right) \delta_{\mathrm{H}}: 8.30(1 \mathrm{H}, \mathrm{bs}) ; 7.56(2 \mathrm{H}, \mathrm{d}, J=7.5 \mathrm{~Hz}) ; 7.27(2 \mathrm{H}, \mathrm{d}, J=7.5 \mathrm{~Hz})$; 7.14$6.95(3 \mathrm{H}, \mathrm{m}) ; 6.32(1 \mathrm{H}, \mathrm{d}, J=8.5 \mathrm{~Hz}) ; 5.05(1 \mathrm{H}, \mathrm{bs}) ; 4.50-4.40(1 \mathrm{H}, \mathrm{m}) ; 4.37(1 \mathrm{H}, \mathrm{dd}, J=8.5 \mathrm{~Hz}, J=5.0$ $\mathrm{Hz}) ; 3.56(3 \mathrm{H}, \mathrm{s}) ; 3.24(1 \mathrm{H}, \mathrm{dd}, J=14.5 \mathrm{~Hz}, J=6.0 \mathrm{~Hz}) ; 3.14(1 \mathrm{H}, \mathrm{dd}, J=14.5 \mathrm{~Hz}, J=7.5 \mathrm{~Hz}) ; 1.91(1 \mathrm{H}$, $\mathrm{m}) ; 1.34(9 \mathrm{H}, \mathrm{s}) ; 0.68(3 \mathrm{H}, \mathrm{d}, J=7.0 \mathrm{~Hz}) ; 0.62(3 \mathrm{H}, \mathrm{d}, J=7.0 \mathrm{~Hz})$.

HPLC retention times 3.93 min.

Diastereomeric excess measured by HPLC using an XTerra MSC8 $5 \mu \mathrm{m} 4.6 \times 250 \mathrm{~mm}$ column. Chromatographic conditions: isocratic elution $95 \% \mathrm{H}_{2} \mathrm{O}\left(0.1 \%\right.$ TFA) : $5 \% \mathrm{CH}_{3} \mathrm{CN}(0.1 \%$ TFA). Retention times (min): 20.35 (major) 21.29 (minor)






\section{Table 1 entry 4}<smiles>CC(=O)[C@H]1CCCN1C(=O)CCCc1ccc([N+](=O)[O-])cc1</smiles>

${ }^{1} \mathrm{H}$ NMR $\left(300 \mathrm{MHz} ; \mathrm{CDCl}_{3}\right.$, major rotamer) $\delta_{\mathrm{H}}: 8.14(2 \mathrm{H}, \mathrm{d}, J=8.5 \mathrm{~Hz}) ; 7.32(2 \mathrm{H}, \mathrm{d}, J=8.5 \mathrm{~Hz}) ; 4.51$ $(1 \mathrm{H}, \mathrm{dd}, J=8.5 \mathrm{~Hz}, J=3.5 \mathrm{~Hz}) ; 3.74(3 \mathrm{H}, \mathrm{s}) ; 3.63-3.54(1 \mathrm{H}, \mathrm{m}) ; 3.50-3.34(1 \mathrm{H}, \mathrm{m}) ; 2.81(2 \mathrm{H}, \mathrm{t}, J=7.5$ $\mathrm{Hz}) ; 2.44-2.30$ (2H, m); 2.24-2.14 (1H, m); 2.11-1.94 (5H, m).

HPLC retention times $3.18 \mathrm{~min}$. ESMS m/z $321.14(\mathrm{M}+\mathrm{H})^{+}$

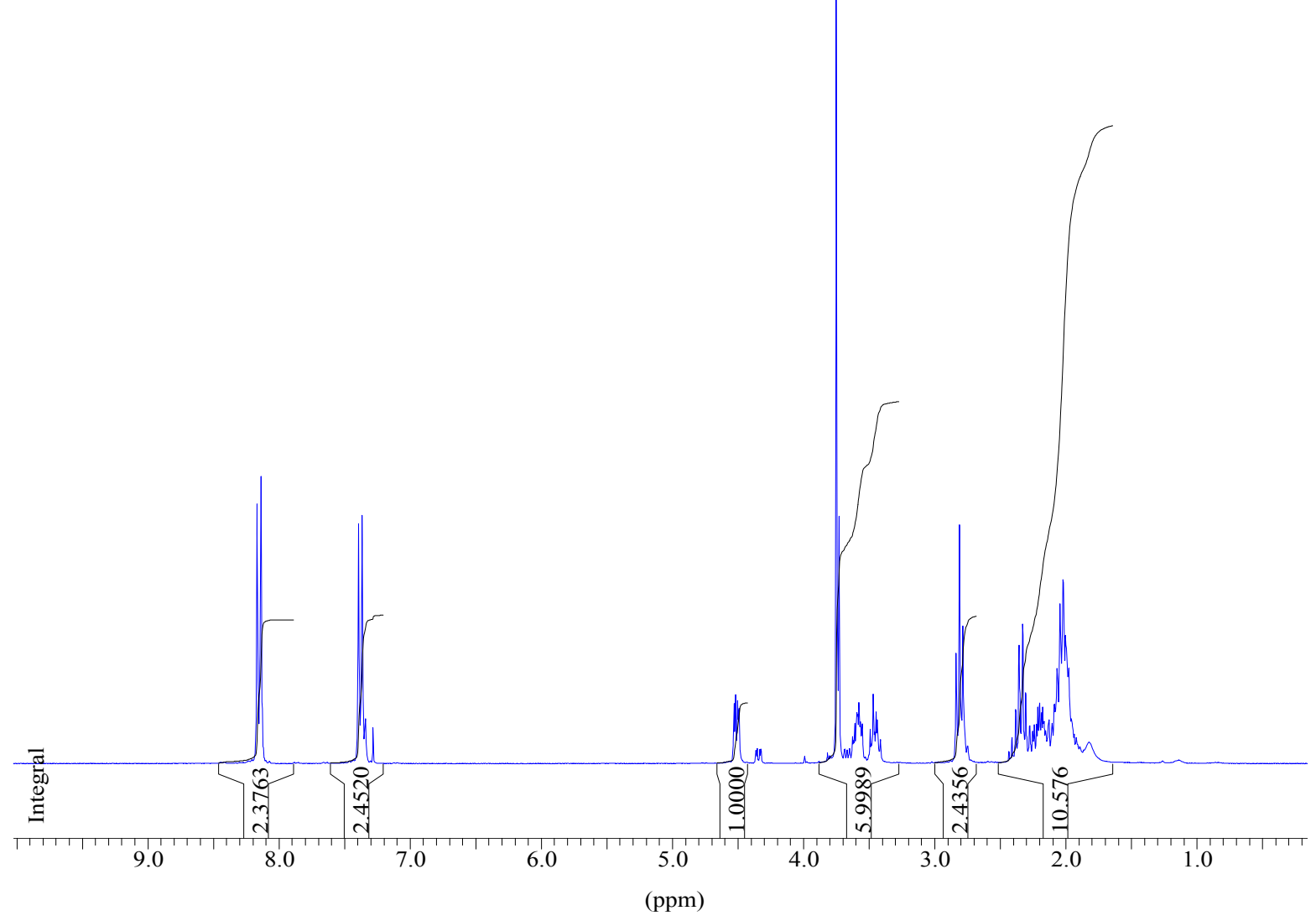




\section{Table 1 entry 5}

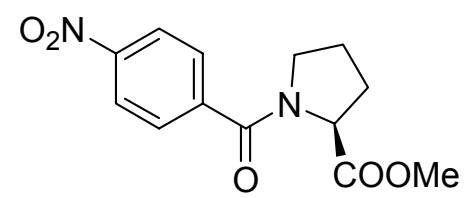

${ }^{1} \mathrm{H}$ NMR $\left(300 \mathrm{MHz} ; \mathrm{CDCl}_{3}\right.$, major rotamer) $\delta_{\mathrm{H}}: 8.21(2 \mathrm{H}, \mathrm{d}, J=8.5 \mathrm{~Hz}) ; 7.67(2 \mathrm{H}, \mathrm{d}, J=8.5 \mathrm{~Hz}) ; 4.61$ $(1 \mathrm{H}, \mathrm{dd}, J=8.5 \mathrm{~Hz}, J=5.0 \mathrm{~Hz}) ; 3.72(3 \mathrm{H}, \mathrm{s}) ; 3.54(1 \mathrm{H}, \mathrm{m}) ; 3.41(1 \mathrm{H}, \mathrm{m}) ; 2.28(1 \mathrm{H}, \mathrm{m}) ; 2.05-1.85(3 \mathrm{H}$, $\mathrm{m})$.

The 2 series of signals visible in the spectrum belong to different rotamers of the same molecule. This has been confirmed by TOCSY experiments and by recording high temperature spectra $\left(80{ }^{\circ} \mathrm{C}\right.$ in $\mathrm{d}_{6^{-}}$ DMSO).

HPLC retention times $2.34 \mathrm{~min}$. ESMS m/z $279.23(\mathrm{M}+\mathrm{H})^{+}$

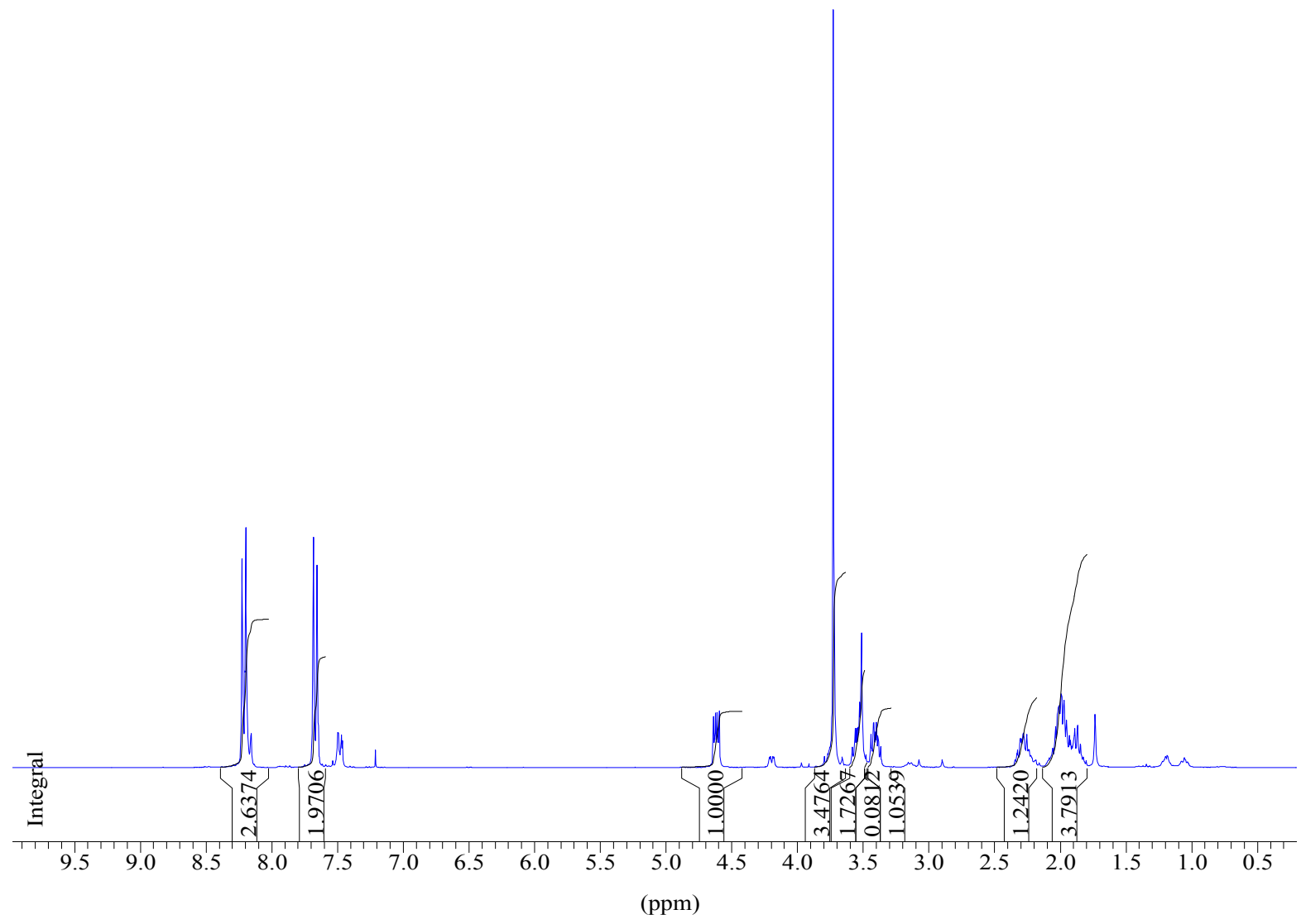




\section{Table 1 entry 6}

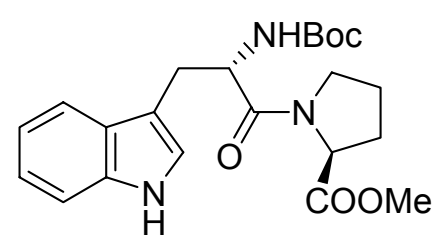

${ }^{1} \mathrm{H}$ NMR (300 MHz; d 6 -DMSO, major rotamer) $\delta_{\mathrm{H}}: 10.87(1 \mathrm{H}, \mathrm{bs}) ; 7.56(2 \mathrm{H}, \mathrm{d}, J=7.5 \mathrm{~Hz}) ; 7.34(2 \mathrm{H}, \mathrm{d}$, $J=7.5 \mathrm{~Hz}) ; 7.22-6.97(3 \mathrm{H}, \mathrm{m}) ; 4.48-4.32(2 \mathrm{H}, \mathrm{m}) ; 3.63(3 \mathrm{H}, \mathrm{s}) ; 3.52-3.42(1 \mathrm{H}, \mathrm{m}) ; 3.34-3.31(1 \mathrm{H}, \mathrm{m})$; 3.08-2.83 (2H, m); 2.23-2.12 (1H, m); 1.98-1.79 (2H, m); $1.31(9 \mathrm{H}, \mathrm{s})$.

HPLC retention times $3.70 \mathrm{~min}$. ESMS m/z $416.22(\mathrm{M}+\mathrm{H})^{+}$

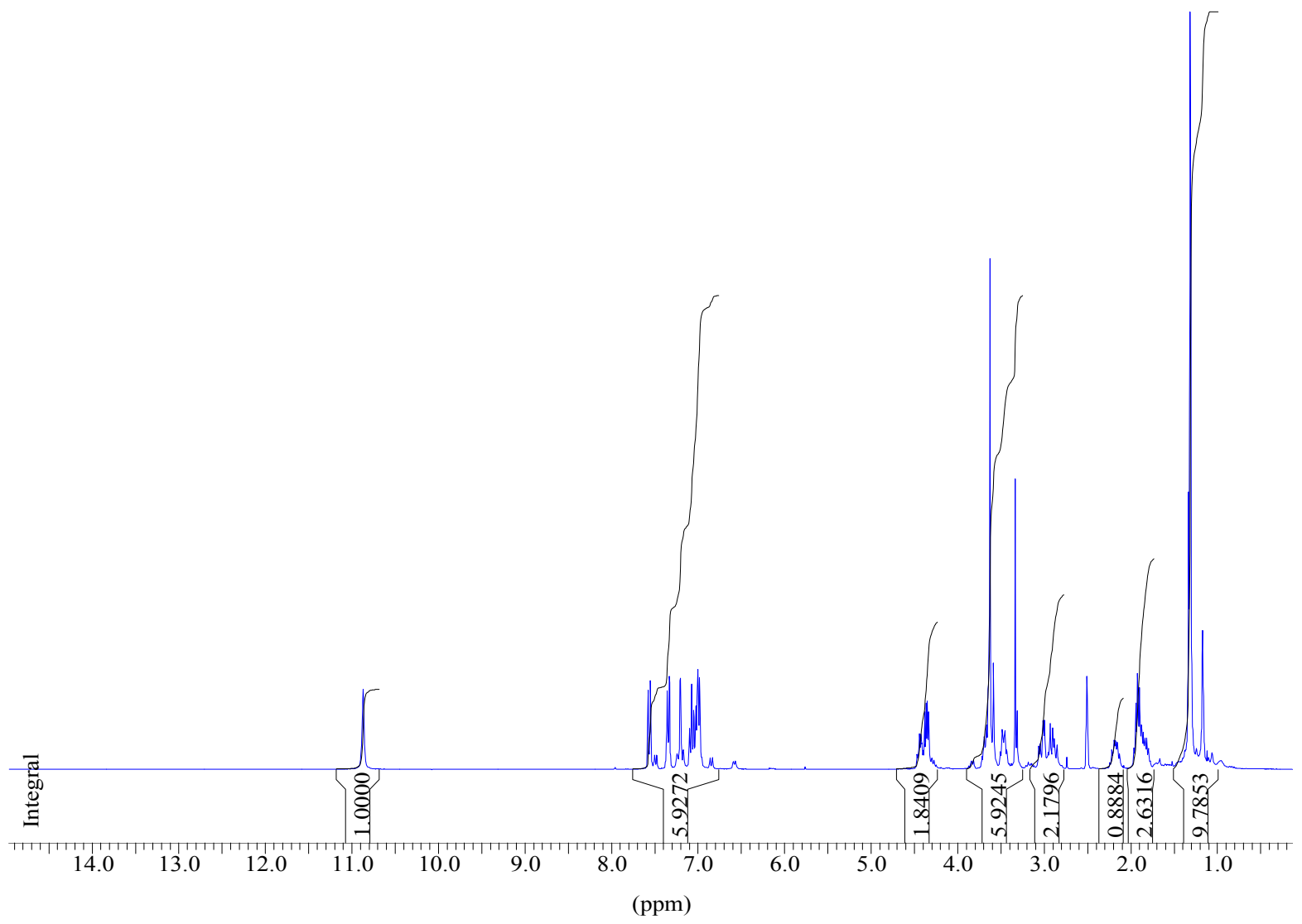




\section{Table 1 entry 8}

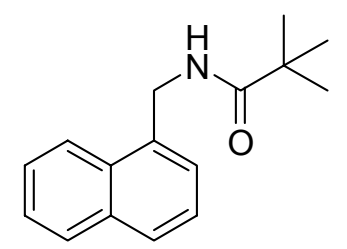

${ }^{1} \mathrm{H}$ NMR $\left(300 \mathrm{MHz} ; \mathrm{CDCl}_{3}\right) \delta_{\mathrm{H}}: 8.02-7.97(1 \mathrm{H}, \mathrm{m}) ; 7.92-7.88(1 \mathrm{H}, \mathrm{m}) ; 7.86-7.81(1 \mathrm{H}, \mathrm{m}) ; 7.59-7.50$ $(2 \mathrm{H}, \mathrm{m}) ; 7.47-7.43(2 \mathrm{H}, \mathrm{m}) ; 5.90(1 \mathrm{H}, \mathrm{bs}) ; 4.90(2 \mathrm{H}, \mathrm{d}, J=5.0 \mathrm{~Hz}) ; 1.23(9 \mathrm{H}, \mathrm{s})$.

HPLC retention times $3.51 \mathrm{~min}$. ESMS m/z $242.29(\mathrm{M}+\mathrm{H})^{+}$

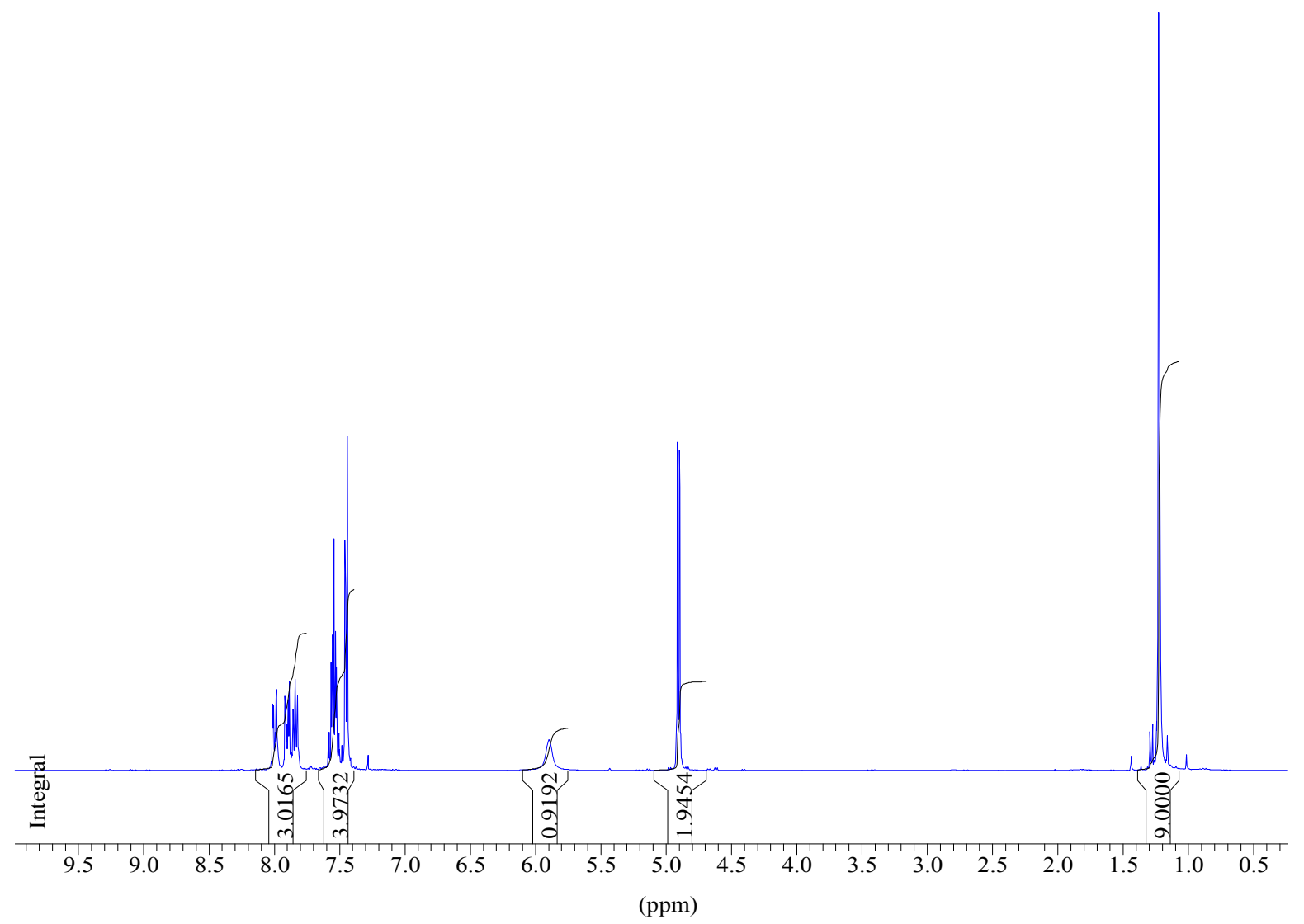




\section{Table 1 entry 9}<smiles>COC(=O)C(Cc1ccc([N+](=O)[O-])cc1)NC(=O)C(C)(C)C</smiles>

${ }^{1} \mathrm{H}$ NMR $\left(300 \mathrm{MHz} ; \mathrm{CDCl}_{3}\right) \delta_{\mathrm{H}}: 8.15(2 \mathrm{H}, \mathrm{d}, J=8.5 \mathrm{~Hz}) ; 7.28(2 \mathrm{H}, \mathrm{d}, J=8.5 \mathrm{~Hz}) ; 6.12(2 \mathrm{H}, \mathrm{d}, J=6.5$ $\mathrm{Hz}) ; 4.90(1 \mathrm{H}, \mathrm{dt}, J=6.5 \mathrm{~Hz}, J=6.0 \mathrm{~Hz}) ; 3.77(3 \mathrm{H}, \mathrm{s}) ; 3.33(1 \mathrm{H}, \mathrm{dd}, J=14.0 \mathrm{~Hz} . J=6.0 \mathrm{~Hz}) ; 1.16(3 \mathrm{H}$, $\mathrm{s})$. HPLC retention time $3.25 \mathrm{~min}$.

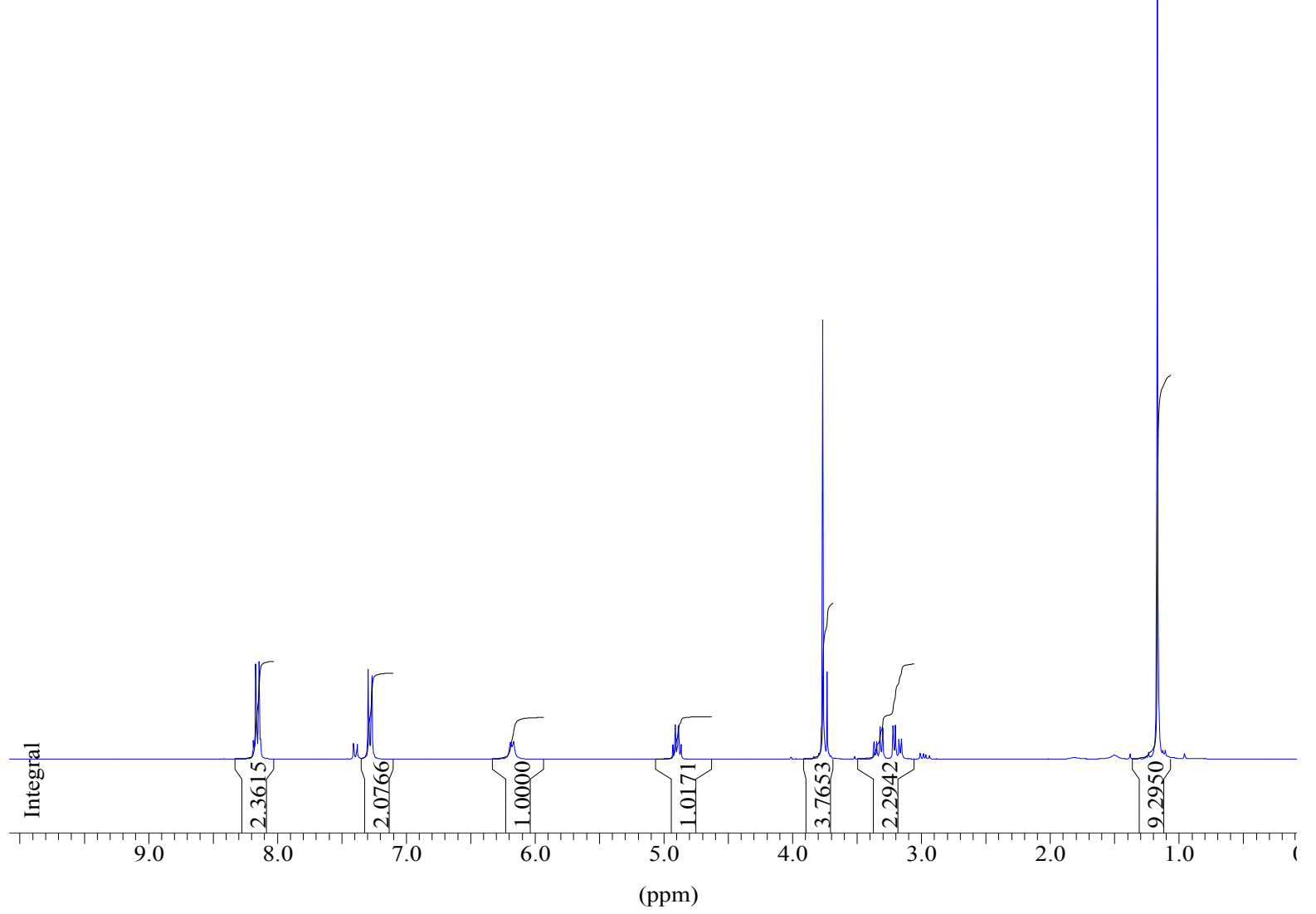




\section{Table 1 entry 10}

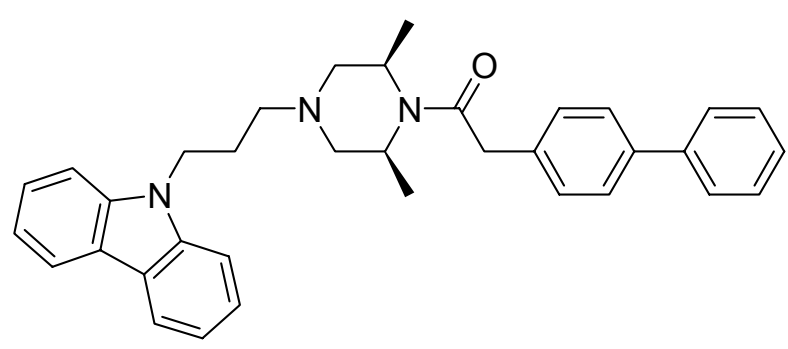

${ }^{1} \mathrm{H}$ NMR $\left(300 \mathrm{MHz} ; \mathrm{CDCl}_{3}\right) \delta_{\mathrm{H}}: 8.01(2 \mathrm{H}, \mathrm{d}, J=8.0 \mathrm{~Hz}) ; 7.48(4 \mathrm{H}, \mathrm{t}, J=8.0 \mathrm{~Hz}) ; 7.39-7.29(6 \mathrm{H}, \mathrm{m})$; 7.26-7.20 (3H, m); 7.17-7.10 (2H, m); $4.61(1 \mathrm{H}, \mathrm{bs}) ; 4.32(2 \mathrm{H}, \mathrm{t}, J=6.5 \mathrm{~Hz}) ; 3.93(1 \mathrm{H}, \mathrm{bs}) ; 3.68(2 \mathrm{H}$, bs), $2.60(2 \mathrm{H}, \mathrm{bs}) ; 2.17(2 \mathrm{H}, \mathrm{t}, J=6.5 \mathrm{~Hz}) ; 1.89(4 \mathrm{H}, \mathrm{m}) ; 1.37(6 \mathrm{H}, \mathrm{t}, J=7.0 \mathrm{~Hz})$.

HPLC retention times $4.03 \mathrm{~min}$. ESMS m/z $516.63(\mathrm{M}+\mathrm{H})^{+}$

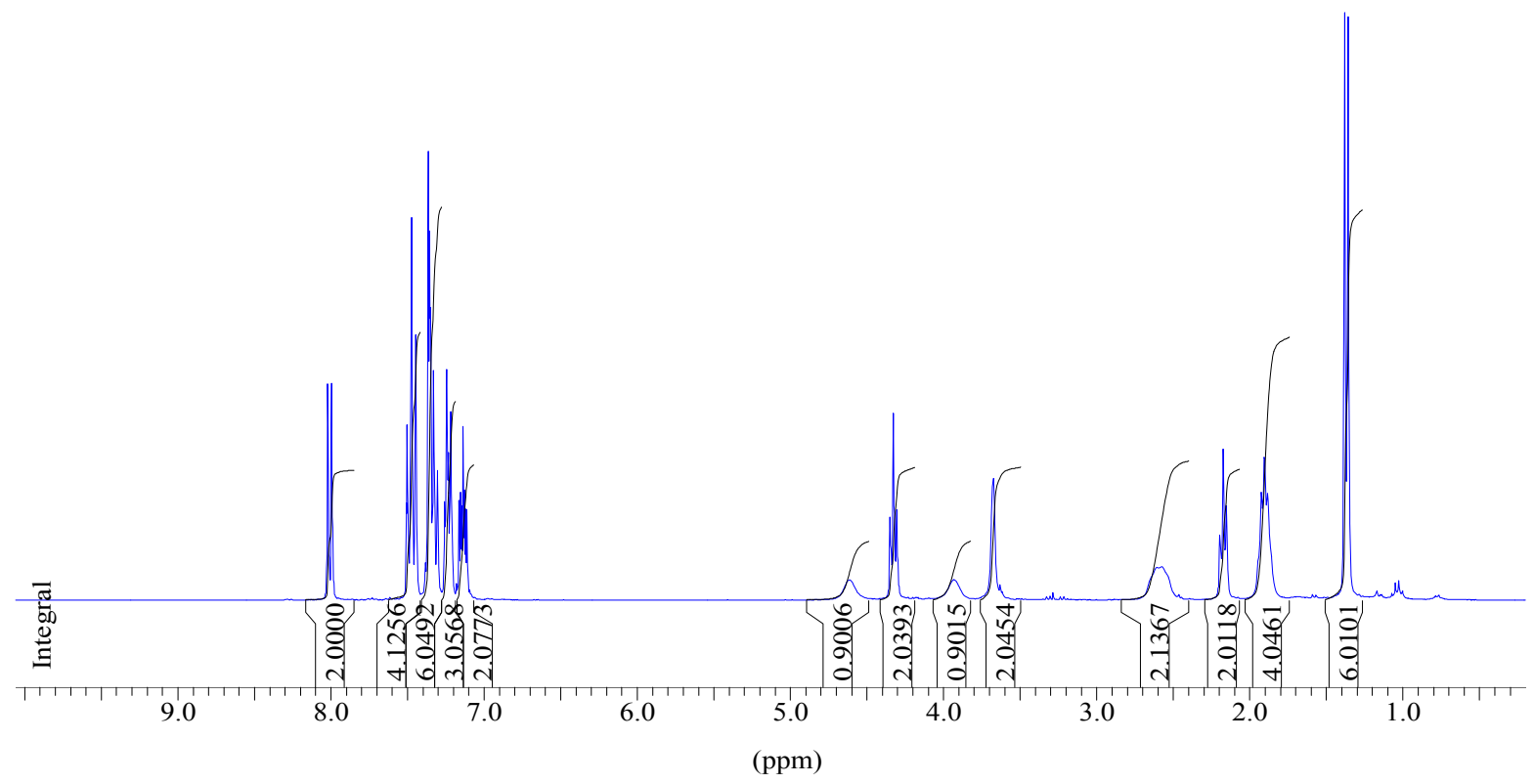




\section{Table 1 entry 11}

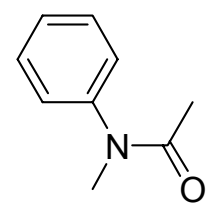

${ }^{1} \mathrm{H}$ NMR $\left(300 \mathrm{MHz} ; \mathrm{CDCl}_{3}\right) \delta_{\mathrm{H}}$ : 7.43-7.31 (3H, m); 7.22-7.17 (2H, m); $3.27(3 \mathrm{H}, \mathrm{s}) ; 1.88(3 \mathrm{H}, \mathrm{s})$. HPLC retention time $1.88 \mathrm{~min}$. ESMS m/z $150.15(\mathrm{M}+\mathrm{H})^{+}$

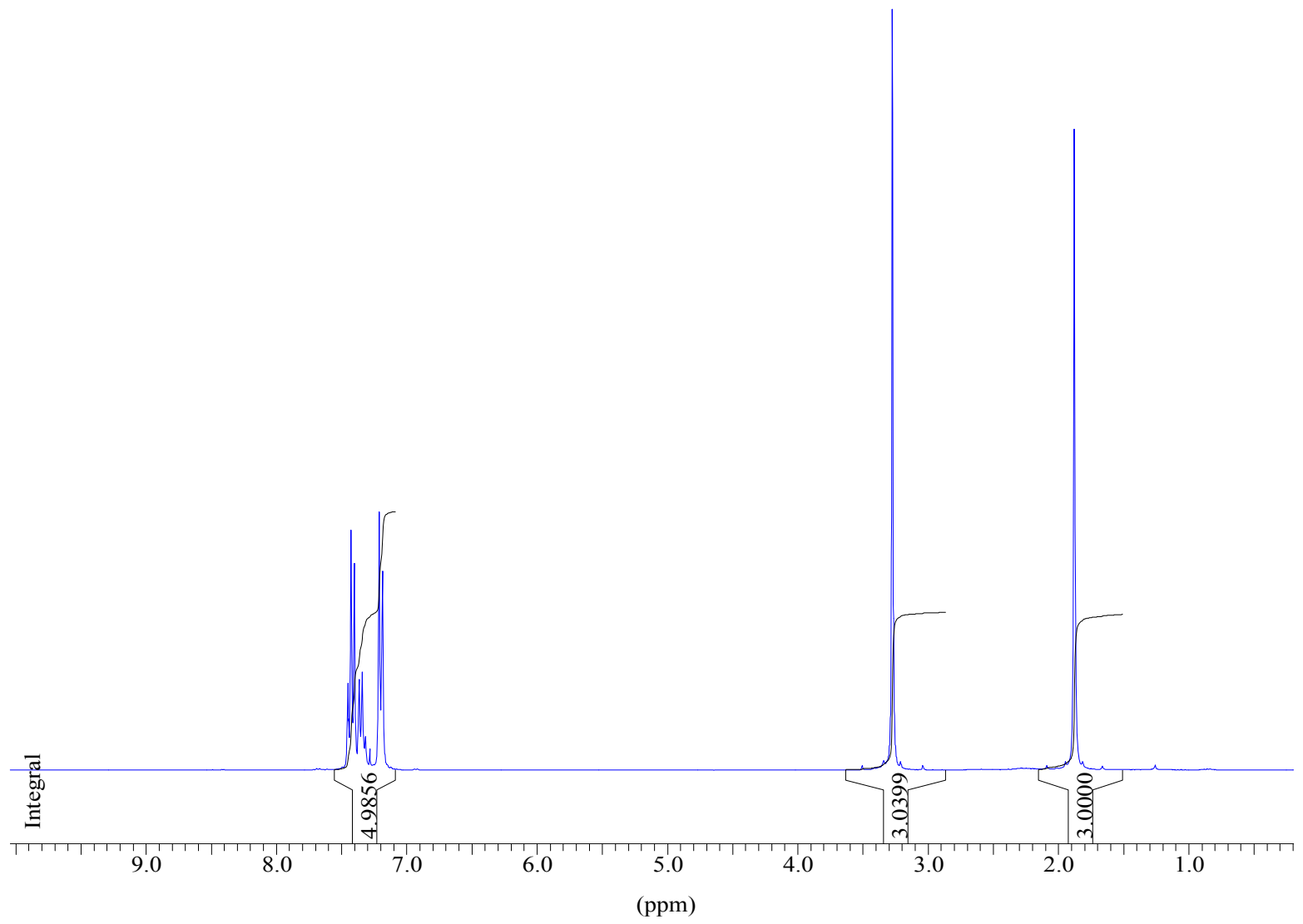




\section{Table 1 entry 12}

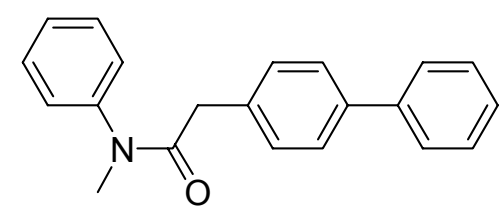

${ }^{1} \mathrm{H}$ NMR $\left(300 \mathrm{MHz} ; \mathrm{CDCl}_{3}\right) \delta_{\mathrm{H}}: 7.61-7.58(2 \mathrm{H}, \mathrm{m}) ; 7.52-7.36(8 \mathrm{H}, \mathrm{m}) ; 7.21-7.17(4 \mathrm{H}, \mathrm{m}) ; 3.54(2 \mathrm{H}, \mathrm{s})$; $3.33(3 \mathrm{H}, \mathrm{s})$.

HPLC retention time $4.34 \mathrm{~min}$. ESMS m/z $302.27(\mathrm{M}+\mathrm{H})^{+}$

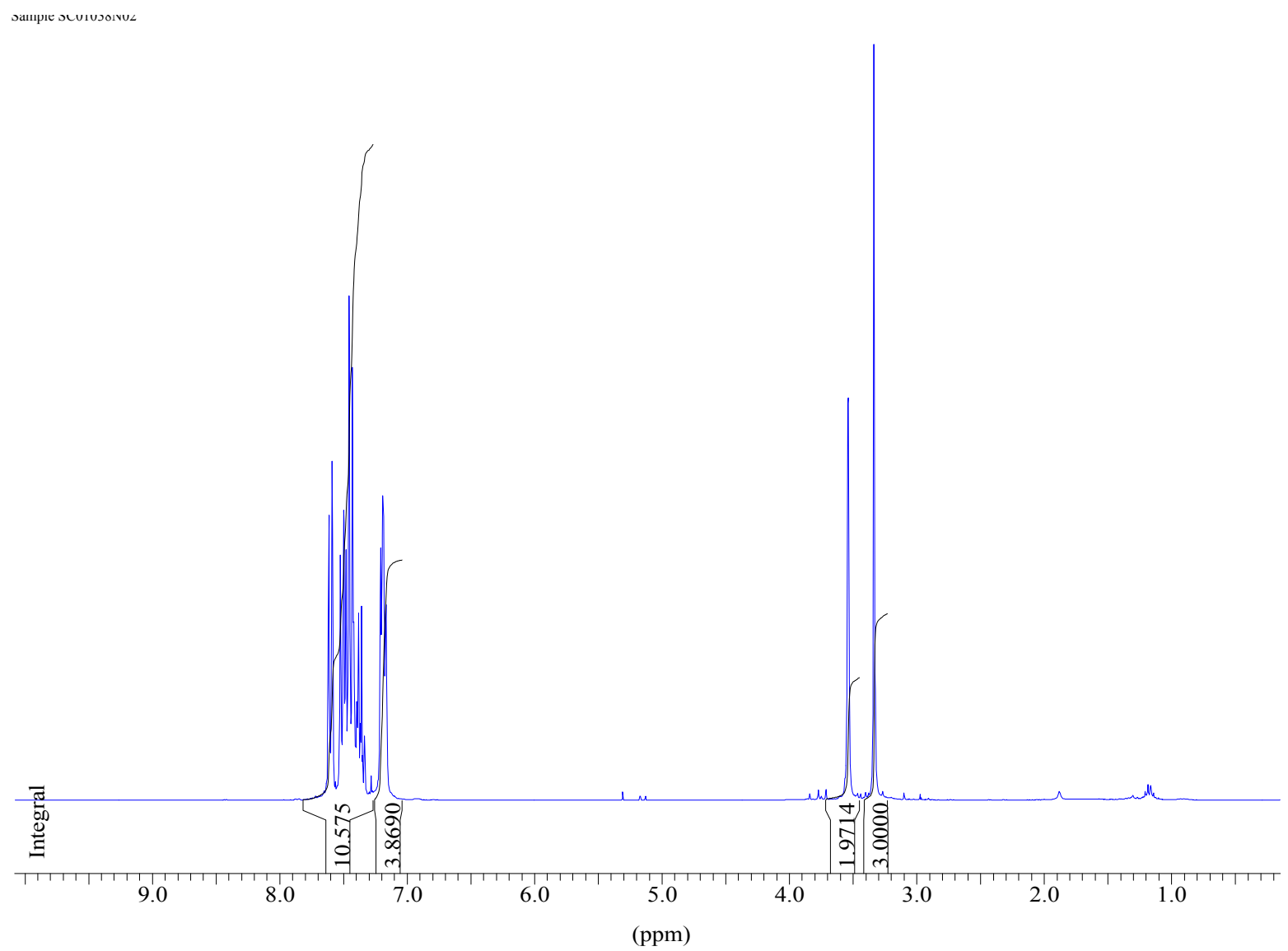




\section{Table 1 entry 13}

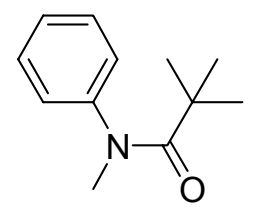

${ }^{1} \mathrm{H}$ NMR $\left(300 \mathrm{MHz} ; \mathrm{CDCl}_{3}\right) \delta_{\mathrm{H}}: 7.35-7.22(3 \mathrm{H}, \mathrm{m}) ; 7.18-7.12(2 \mathrm{H}, \mathrm{m}) ; 3.14(3 \mathrm{H}, \mathrm{s}) ; 0.97$ (9H, s). HPLC retention time $3.29 \mathrm{~min}$. ESMS m/z $192.14(\mathrm{M}+\mathrm{H})^{+}$

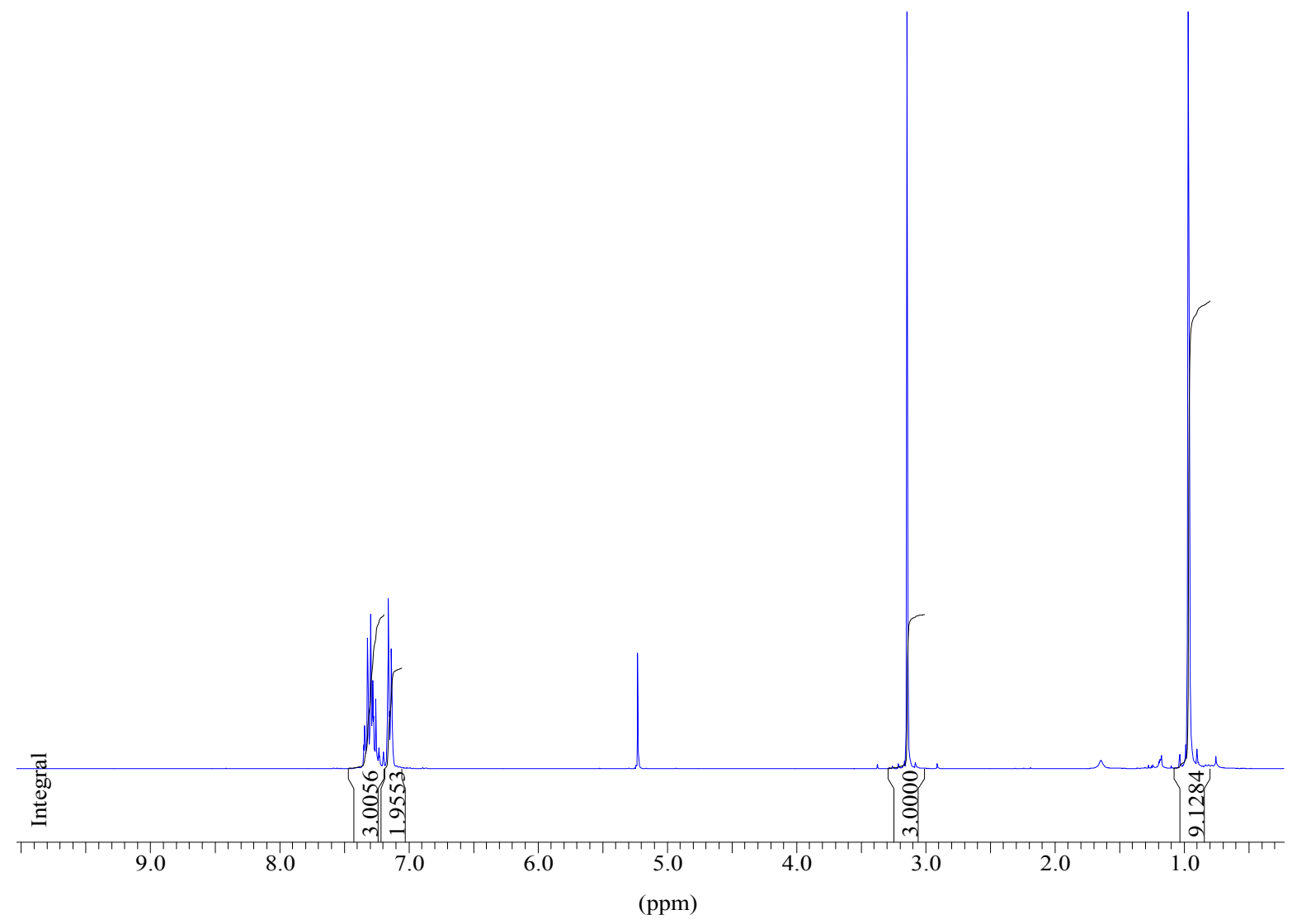




\section{Table 1 entry 14}<smiles>COC(=O)C(Cc1ccc([N+](=O)[O-])cc1)NC=O</smiles>

${ }^{1} \mathrm{H}$ NMR (300 MHz; d $\mathrm{d}_{6}$-DMSO) $\delta_{\mathrm{H}}: 8.16(2 \mathrm{H}, \mathrm{d}, J=8.5 \mathrm{~Hz}) ; 7.99(1 \mathrm{H}, \mathrm{s}) ; 7.52(2 \mathrm{H}, \mathrm{d}, J=8.5 \mathrm{~Hz}) ; 4.70$ $(1 \mathrm{H}, \mathrm{m}) ; 3.65(3 \mathrm{H}, \mathrm{s}) ; 3.24(1 \mathrm{H}, \mathrm{dd}, J=13.5 \mathrm{~Hz} . J=5.5 \mathrm{~Hz}) ; 3.06(1 \mathrm{H}, \mathrm{dd}, J=13.5 \mathrm{~Hz} . J=9.5 \mathrm{~Hz})$. HPLC retention time $2.00 \mathrm{~min}$. ESMS m/z $251.2(\mathrm{M}-\mathrm{H})^{-}$

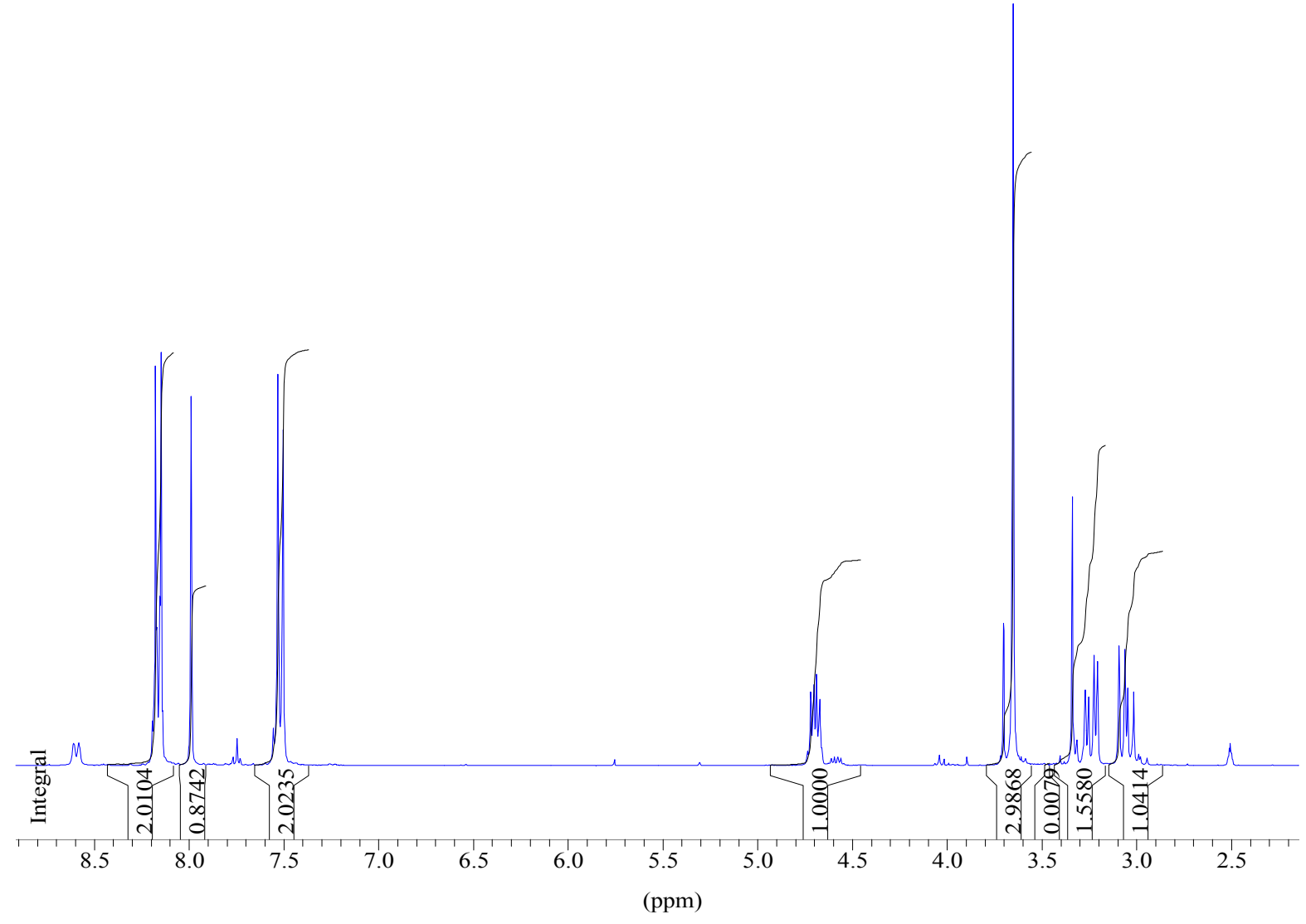


Table 2 entry 1

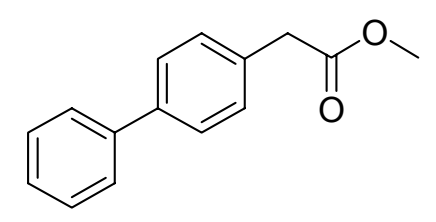

${ }^{1} \mathrm{H}$ NMR $\left(300 \mathrm{MHz} ; \mathrm{CDCl}_{3}\right) \delta_{\mathrm{H}}: 7.66-7.58(4 \mathrm{H}, \mathrm{m}) ; 7.51-7.44(2 \mathrm{H}, \mathrm{m}) ; 7.43-7.35(3 \mathrm{H}, \mathrm{m}) ; 3.76(3 \mathrm{H}, \mathrm{s})$; $3.72(2 \mathrm{H}, \mathrm{s})$.

HPLC retention time $4.02 \mathrm{~min}$.

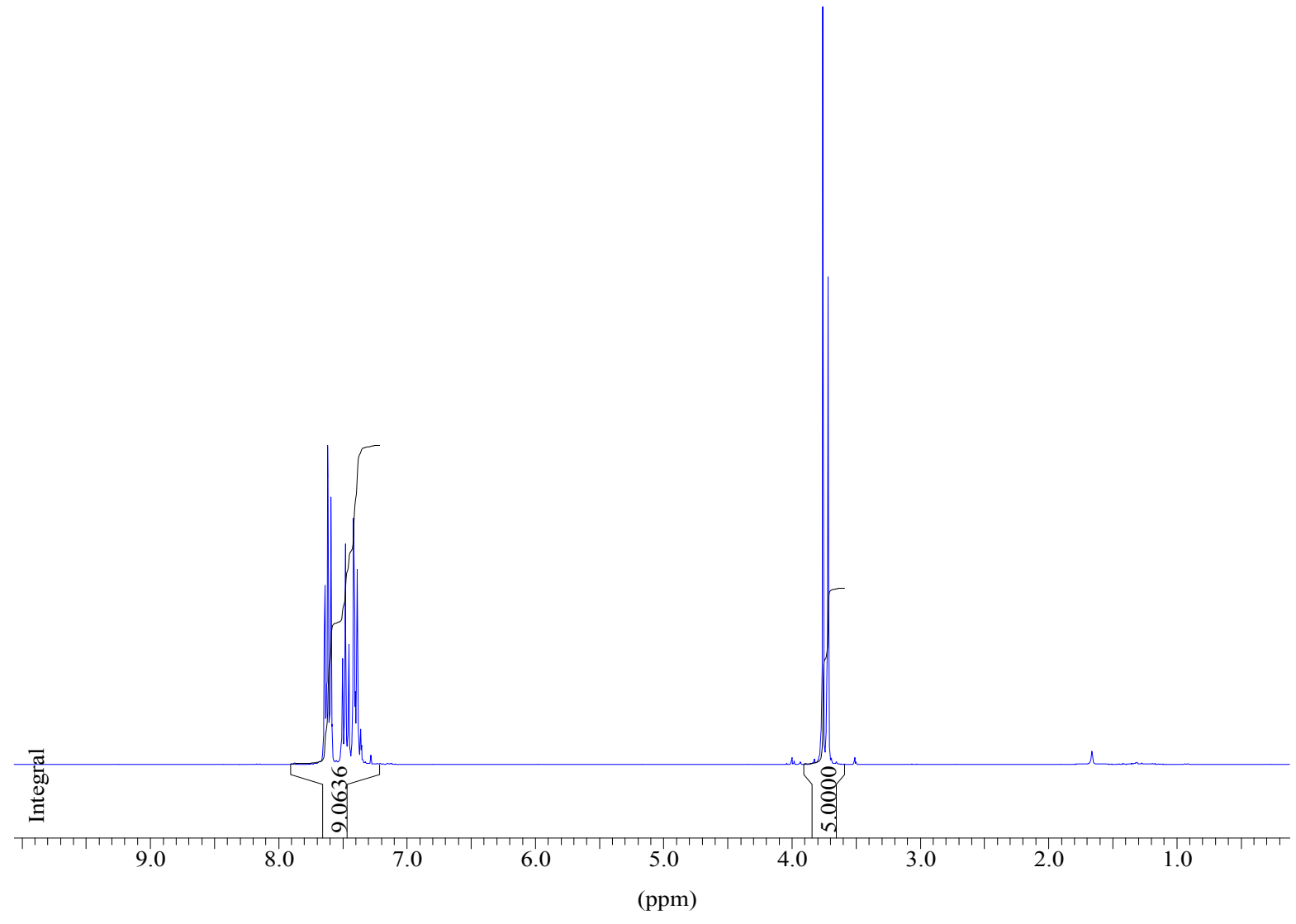




\section{Table 2 entry 2}

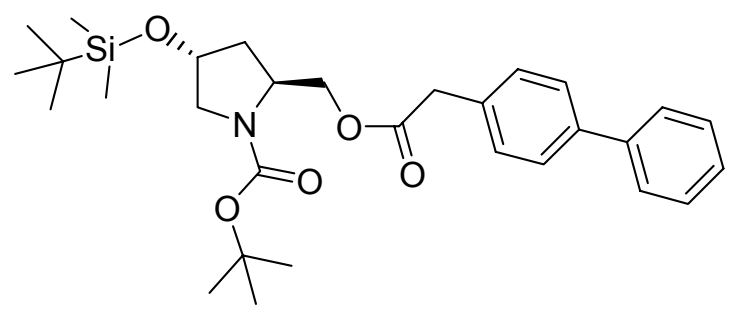

${ }^{1} \mathrm{H}$ NMR $\left(300 \mathrm{MHz} ; \mathrm{CDCl}_{3}\right) \delta_{\mathrm{H}}: 7.58-7.49(4 \mathrm{H}, \mathrm{m}) ; 7.44-7.36(2 \mathrm{H}, \mathrm{m}) ; 7.35-7.27$ (3H, m); 4.30-4.20 $(2 \mathrm{H}, \mathrm{m}) ; 4.19-4.00(2 \mathrm{H}, \mathrm{m}) ; 3.64(2 \mathrm{H}, \mathrm{s}) ; 3.56-3.18(3 \mathrm{H}, \mathrm{m}) ; 1.97-1.75(2 \mathrm{H}, \mathrm{m}) ; 1.42(9 \mathrm{H}, \mathrm{s}), 0.83(9 \mathrm{H}$, s); $0.04(3 \mathrm{H}, \mathrm{s}) ; 0.01(3 \mathrm{H}, \mathrm{s})$.

HPLC retention time $6.33 \mathrm{~min}$. ESMS m/z $426.4(\mathrm{M}+\mathrm{H}-\mathrm{Boc})^{+}$

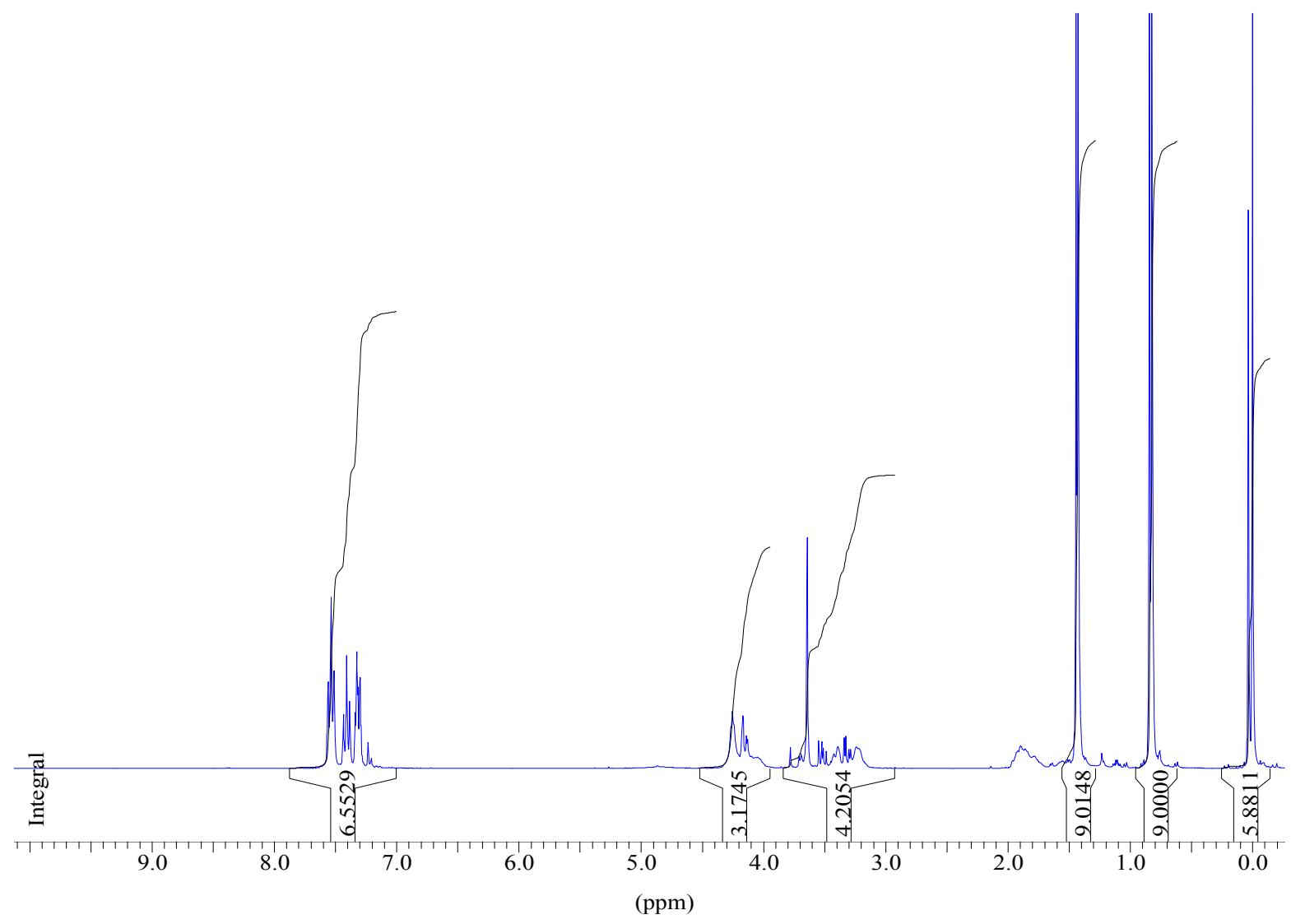




\section{Table 2 entry 3}

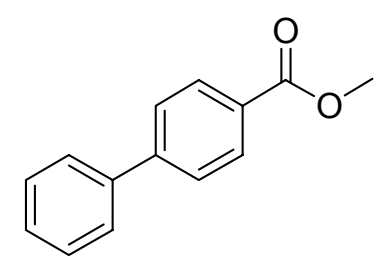

${ }^{1} \mathrm{H}$ NMR $\left(300 \mathrm{MHz} ; \mathrm{CDCl}_{3}\right) \delta_{\mathrm{H}}: 8.13(2 \mathrm{H}, \mathrm{d}, J=6.5 \mathrm{~Hz}) ; 7.70-7.64(4 \mathrm{H}, \mathrm{m}) ; 7.52-7.39$ (3H, m); 3.97 $(3 \mathrm{H}, \mathrm{s})$.

HPLC retention time $4.04 \mathrm{~min}$. ESMS m/z $213.3(\mathrm{M}+\mathrm{H})^{+}$

Sallpie sevivacive

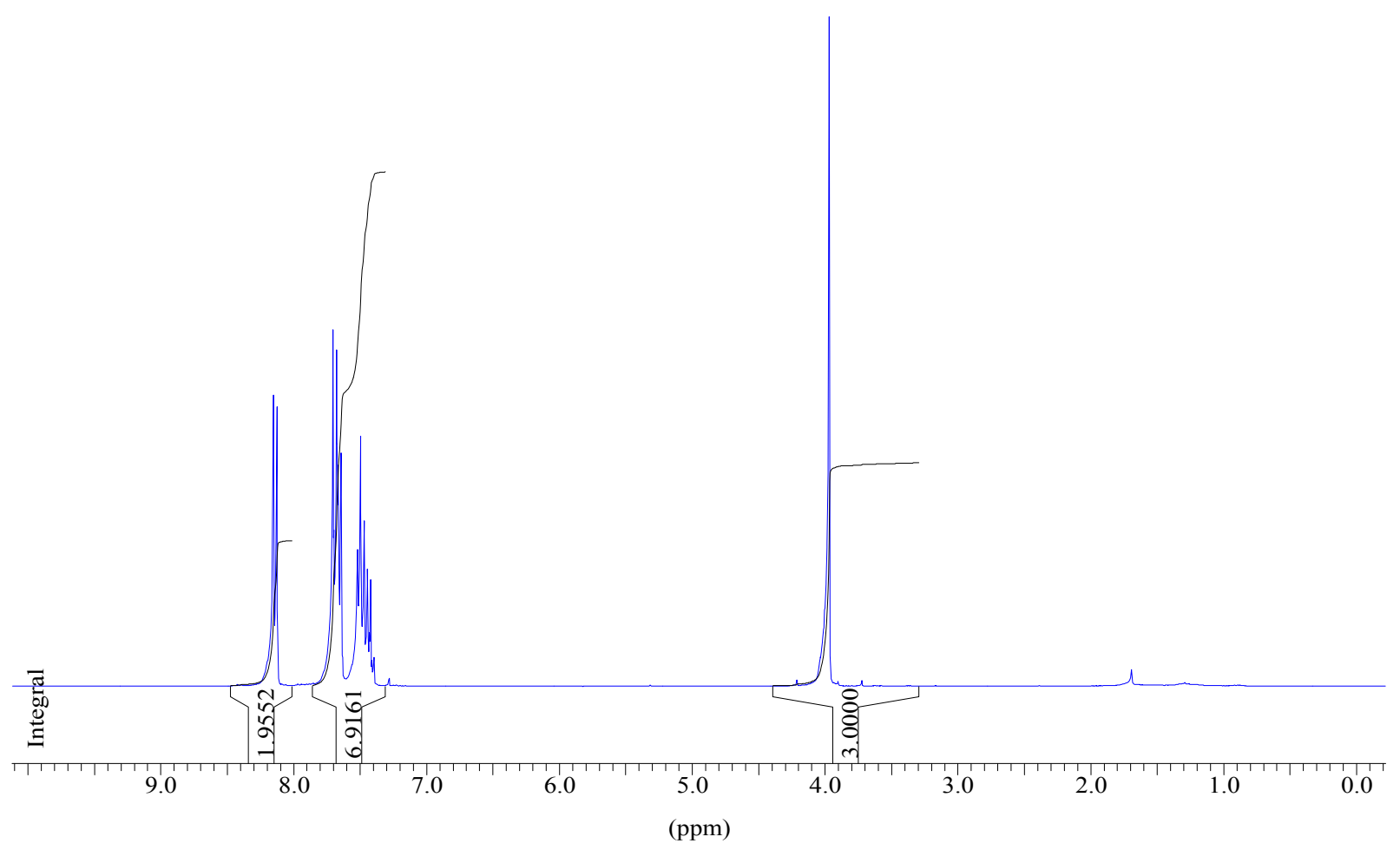




\section{Table 2 entry 4}

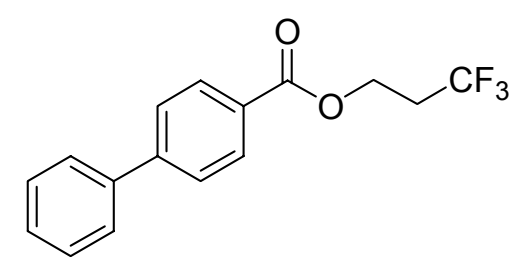

${ }^{1} \mathrm{H}$ NMR $\left(300 \mathrm{MHz} ; \mathrm{CDCl}_{3}\right) \delta_{\mathrm{H}}: 8.17(2 \mathrm{H}, \mathrm{d}, J=8.5 \mathrm{~Hz}) ; 7.75-7.70(2 \mathrm{H}, \mathrm{m}) ; 7.68-7.63$ (2H, m); 7.54$7.41(3 \mathrm{H}, \mathrm{m}) ; 4.75(2 \mathrm{H}, \mathrm{q}, J=8.5 \mathrm{~Hz})$.

HPLC retention time $4.88 \mathrm{~min}$.

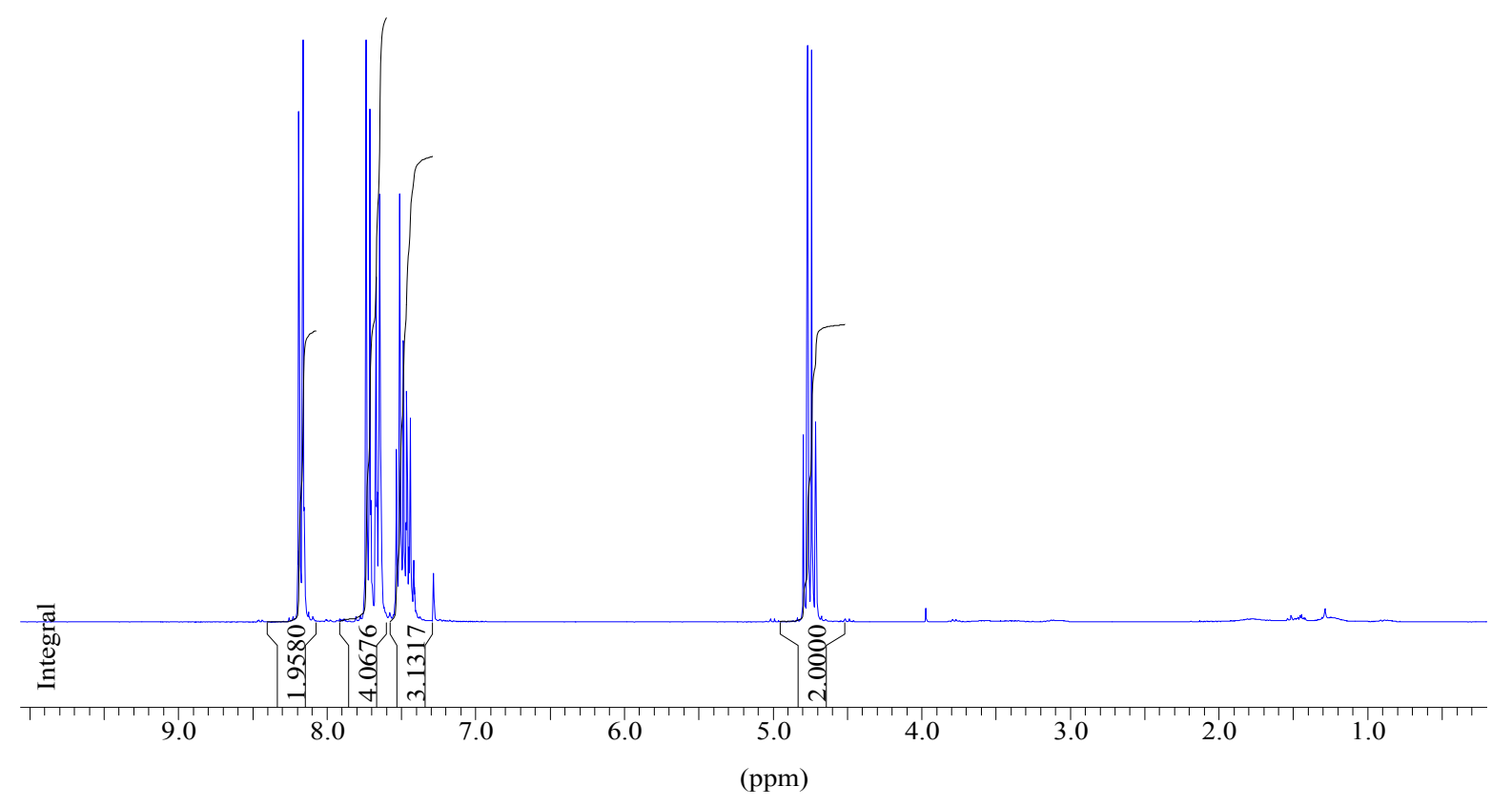




\section{Table 2 entry 5}

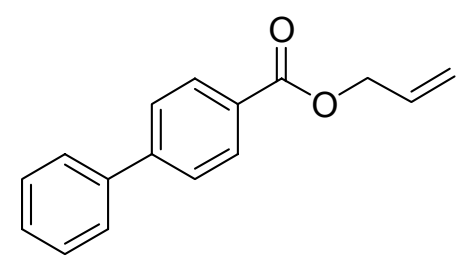

${ }^{1} \mathrm{H}$ NMR $\left(300 \mathrm{MHz} ; \mathrm{CDCl}_{3}\right) \delta_{\mathrm{H}}: 8.05(1 \mathrm{H}, \mathrm{d}, J=8.5 \mathrm{~Hz}) ; 7.61-7.51(4 \mathrm{H}, \mathrm{m}) ; 7.42-7.28(3 \mathrm{H}, \mathrm{m}) ; 5.98$ $(1 \mathrm{H}, \mathrm{ddt}, J=17.5 \mathrm{~Hz}, 10.0 \mathrm{~Hz}, 6.0 \mathrm{~Hz}) ; 5.36(1 \mathrm{H}, \mathrm{dd}, J=17.5 \mathrm{~Hz}, 1.5 \mathrm{~Hz}) ; 5.26(1 \mathrm{H}, \mathrm{dd}, J=10.0 \mathrm{~Hz}, 1.5$ $\mathrm{Hz}) ; 4.77(2 \mathrm{H}, \mathrm{d}, J=6.0 \mathrm{~Hz})$.

HPLC retention time $4.60 \mathrm{~min}$.

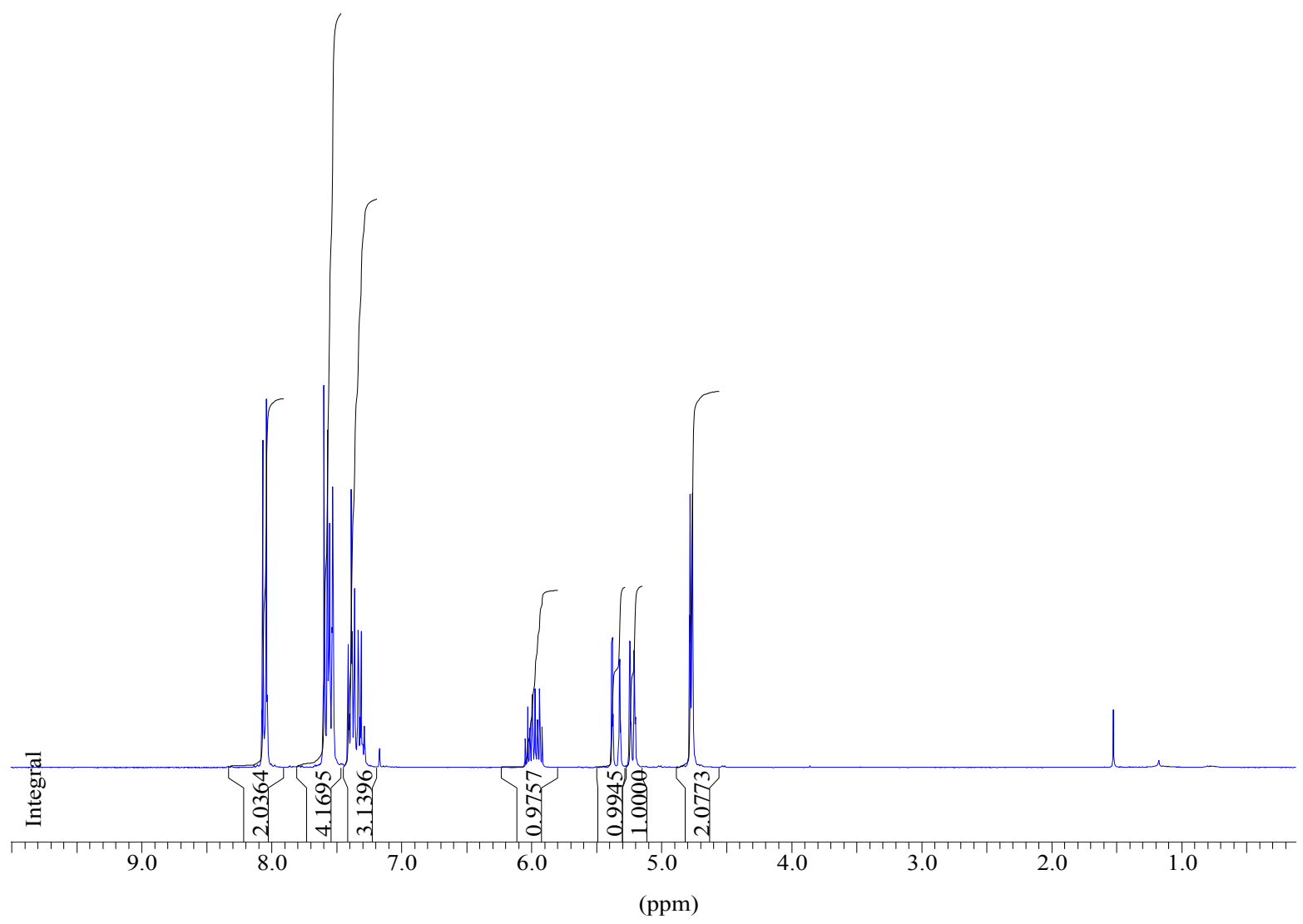




\section{Table $\underline{2}$ entry $\underline{6}$}

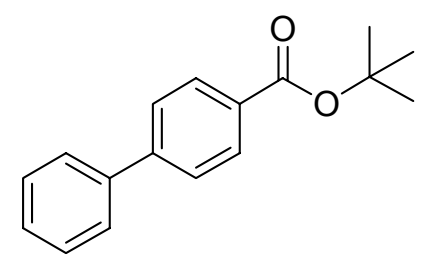

${ }^{1} \mathrm{H}$ NMR $\left(300 \mathrm{MHz} ; \mathrm{CDCl}_{3}\right) \delta_{\mathrm{H}}: 8.08(2 \mathrm{H}, \mathrm{d}, J=7.5 \mathrm{~Hz}) ; 7.68-7.63(4 \mathrm{H}, \mathrm{m}) ; 7.52-7.41(3 \mathrm{H}, \mathrm{m}) ; 1.65$ (9H, s).

HPLC retention time $4.83 \mathrm{~min}$.

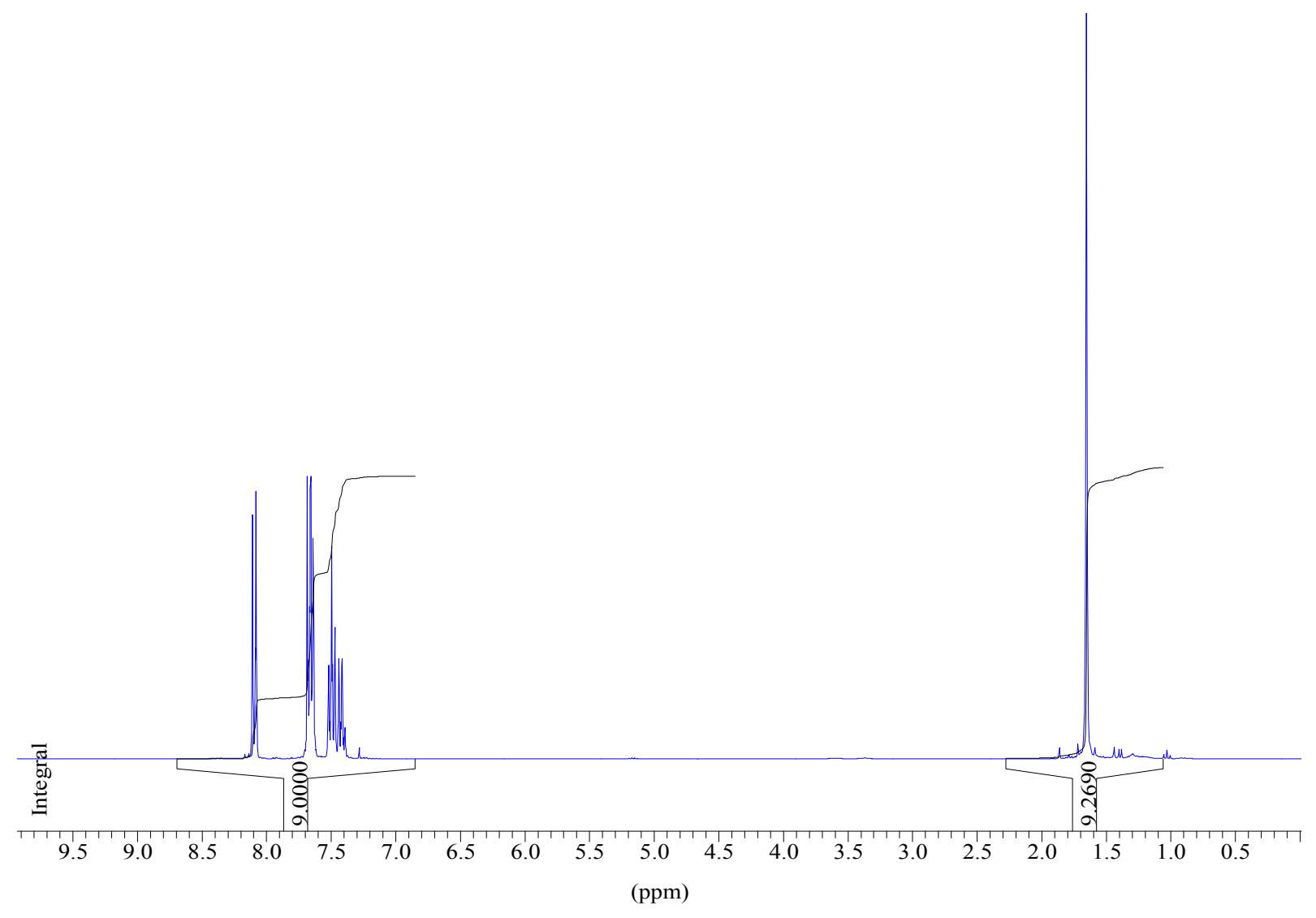




\section{Table 2 entry 7}

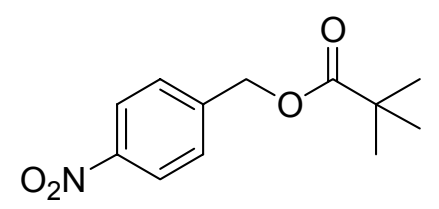

${ }^{1} \mathrm{H}$ NMR $\left(300 \mathrm{MHz} ; \mathrm{CDCl}_{3}\right) \delta_{\mathrm{H}}: 8.24(2 \mathrm{H}, \mathrm{d}, J=8.5 \mathrm{~Hz}) ; 7.52(2 \mathrm{H}, \mathrm{d}, J=8.5 \mathrm{~Hz}) ; 5.51(2 \mathrm{H}, \mathrm{s}) ; 1.27$ $(9 \mathrm{H}, \mathrm{s})$.

HPLC retention time 3.96 min.

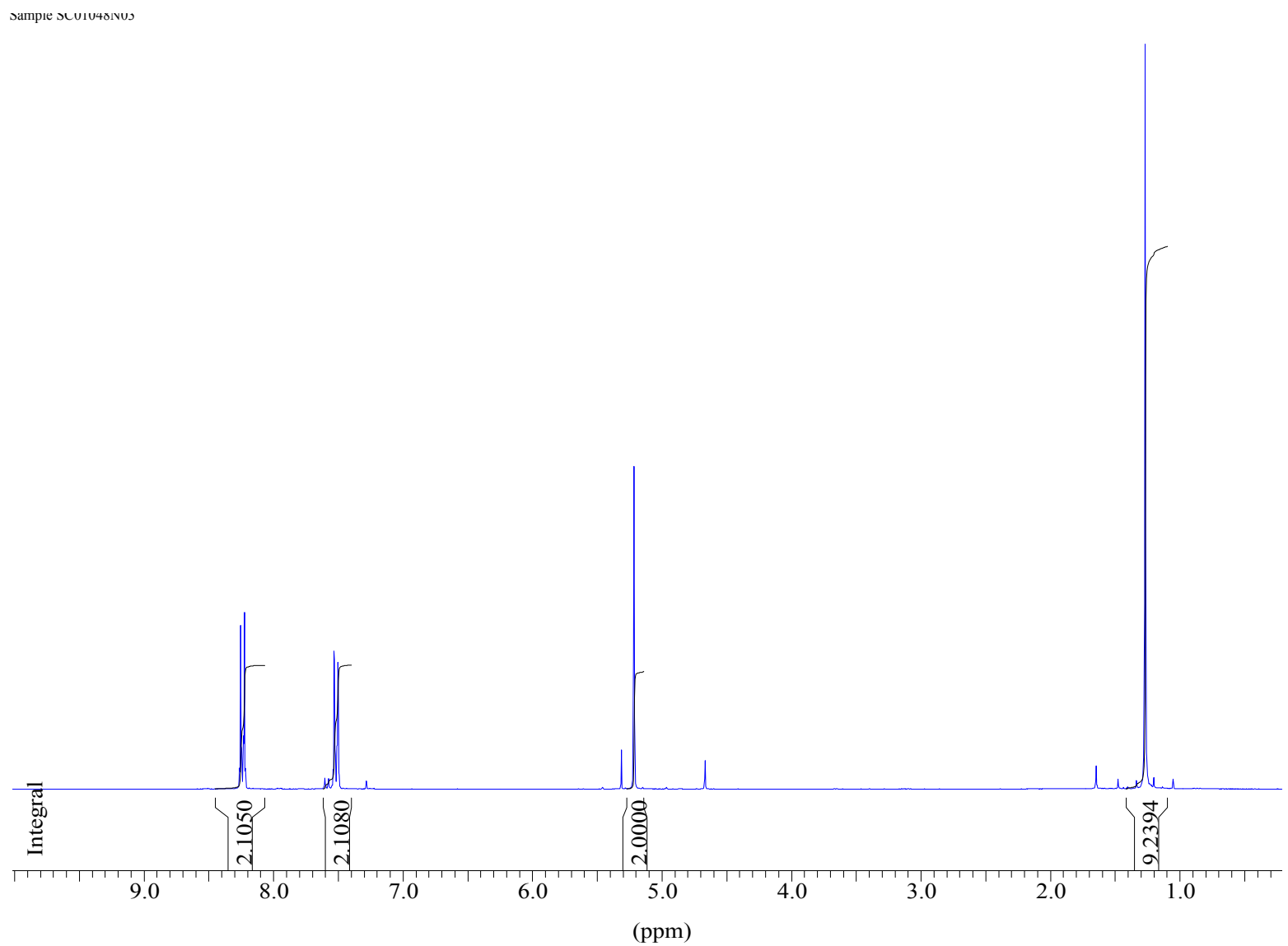


Table 2 entry 8<smiles>O=C(CCc1ccc([N+](=O)[O-])cc1)OC(C(=O)c1ccccc1)c1ccccc1</smiles>

${ }^{1} \mathrm{H}$ NMR $\left(300 \mathrm{MHz} ; \mathrm{CDCl}_{3}\right) \delta_{\mathrm{H}}: 8.07(2 \mathrm{H}, \mathrm{d}, J=8.5 \mathrm{~Hz}) ; 7.86(2 \mathrm{H}, \mathrm{m}) ; 7.46(1 \mathrm{H}, \mathrm{m}) ; 7.39-7.27(9 \mathrm{H}$, $\mathrm{m}) ; 6.80$ (1H, bs); $3.06(2 \mathrm{H}, \mathrm{d}, J=7.5 \mathrm{~Hz}) ; 2.91-2.70(2 \mathrm{H}, \mathrm{m})$.

HPLC retention time $4.79 \mathrm{~min}$.

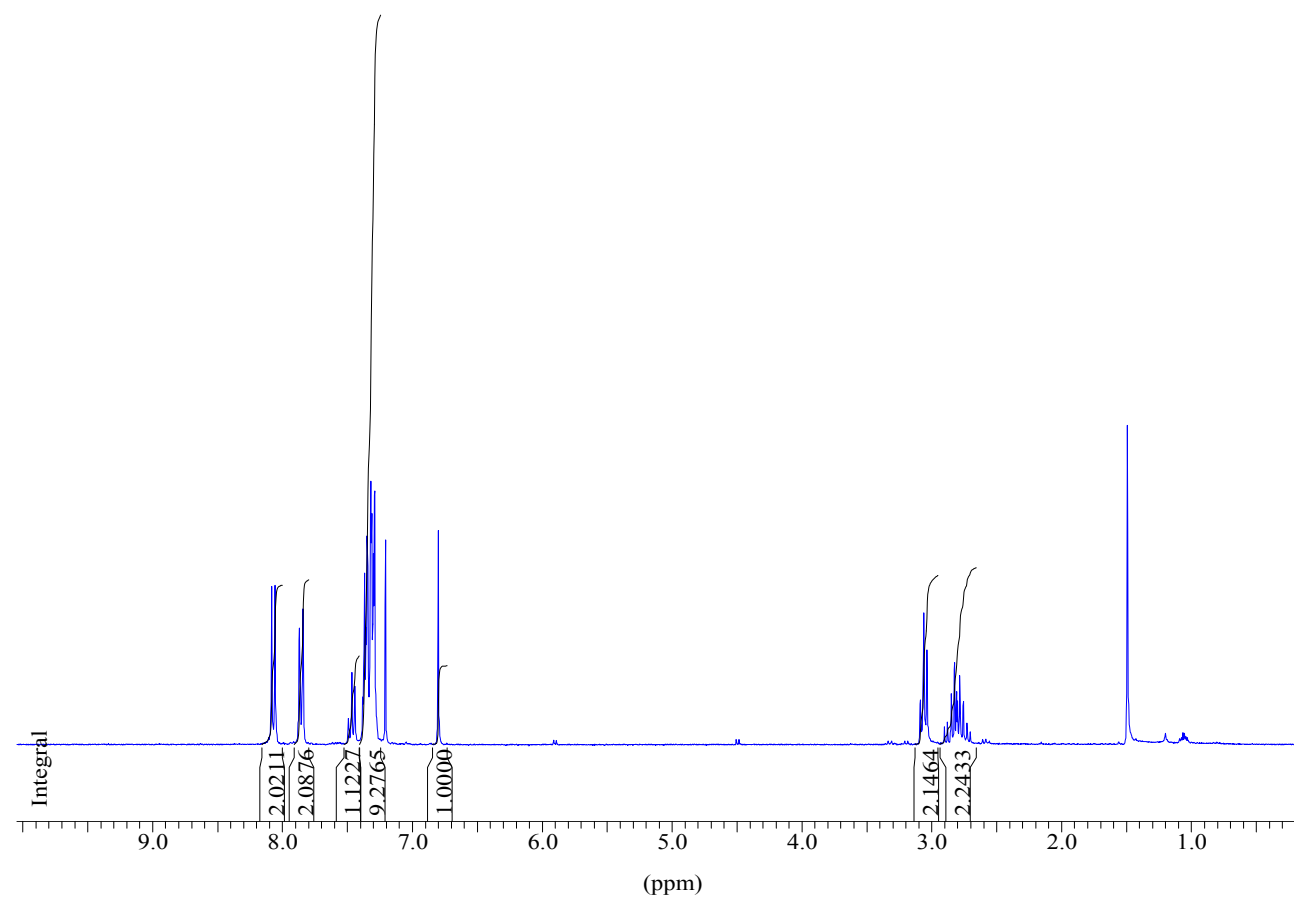




\section{Table 2 entry 9}<smiles>CO[C@](C(=O)OC(C(=O)c1ccccc1)c1ccccc1)(c1ccccc1)C(F)(F)F</smiles>

Starting from racemic benzoin, a mixture of 2 diastereoisomers was obtained. The ratio could be determined using either the ${ }^{1} \mathrm{H}$ spectrum (two separated singlets for the $\mathrm{OCH} 3$ resonance at 3.78 and $3.59 \mathrm{ppm}$ ) or the ${ }^{19} \mathrm{~F}$ spectrum (signals at -72.2 and $-72.7 \mathrm{ppm}$ ).

HPLC retention time 5.14.

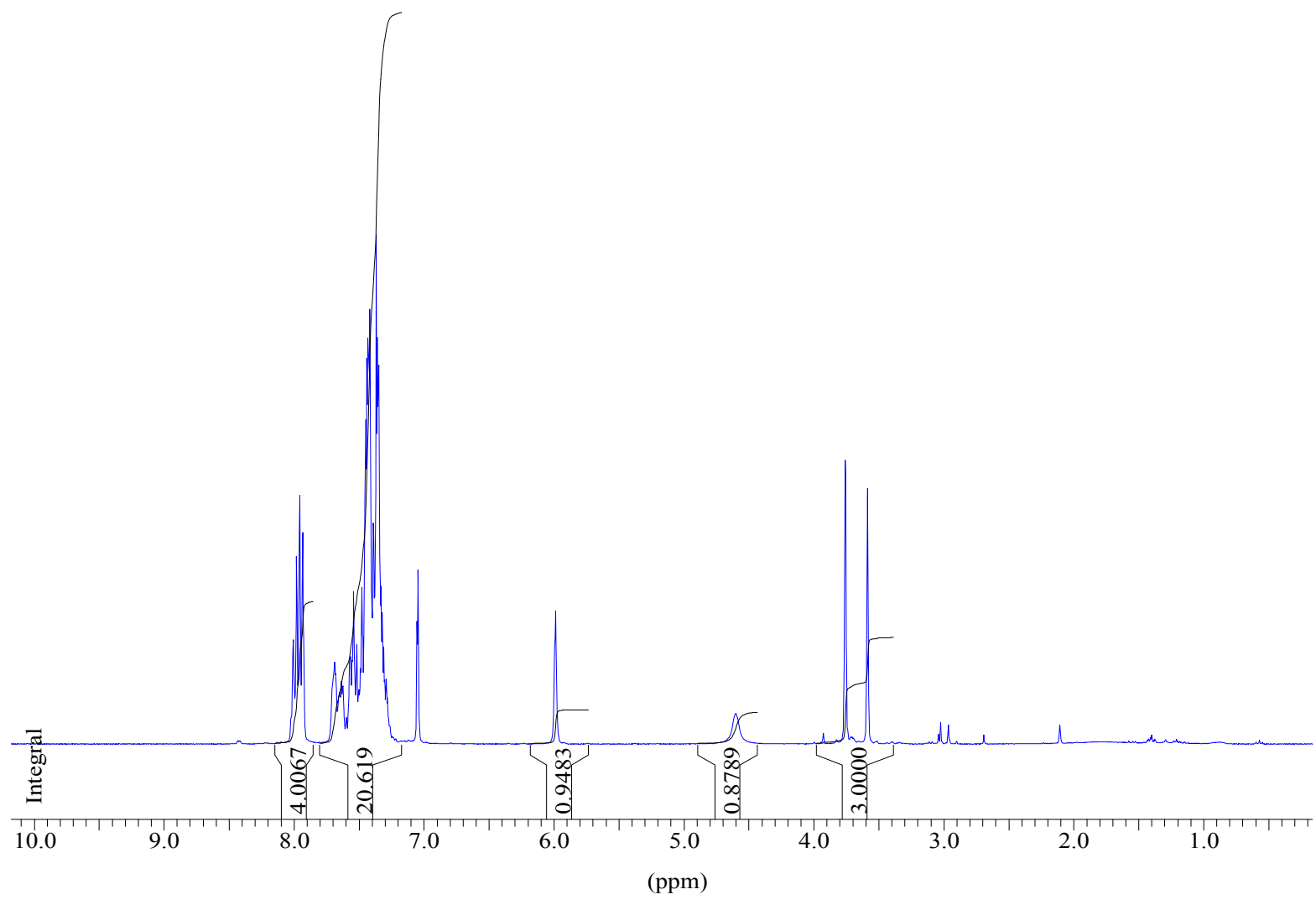




\section{Table 2 entry 10}

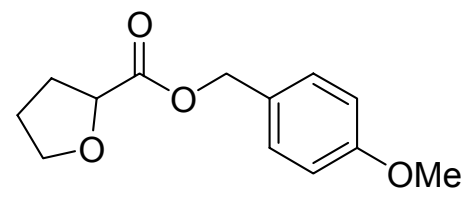

${ }^{1} \mathrm{H}$ NMR $\left(300 \mathrm{MHz} ; \mathrm{CDCl}_{3}\right) \delta_{\mathrm{H}}: 7.31(2 \mathrm{H}, \mathrm{d}, J=8.5 \mathrm{~Hz}) ; 6.90(2 \mathrm{H}, \mathrm{d}, J=8.5 \mathrm{~Hz}) ; 5.15(1 \mathrm{H}, \mathrm{d}, J=12.0$ $\mathrm{Hz}) ; 5.10(2 \mathrm{H}, \mathrm{d}, J=12.0 \mathrm{~Hz}) ; 4.47(1 \mathrm{H}, \mathrm{dd}, J=8.0 \mathrm{~Hz}, J=4.5 \mathrm{~Hz}) ; 4.08-3.89(2 \mathrm{H}, \mathrm{m}) ; 3.81(3 \mathrm{H}, \mathrm{s})$; 2.30-2.19 (1H, m); 2.07-1.87 (3H, m).

HPLC retention time $3.04 \mathrm{~min}$.

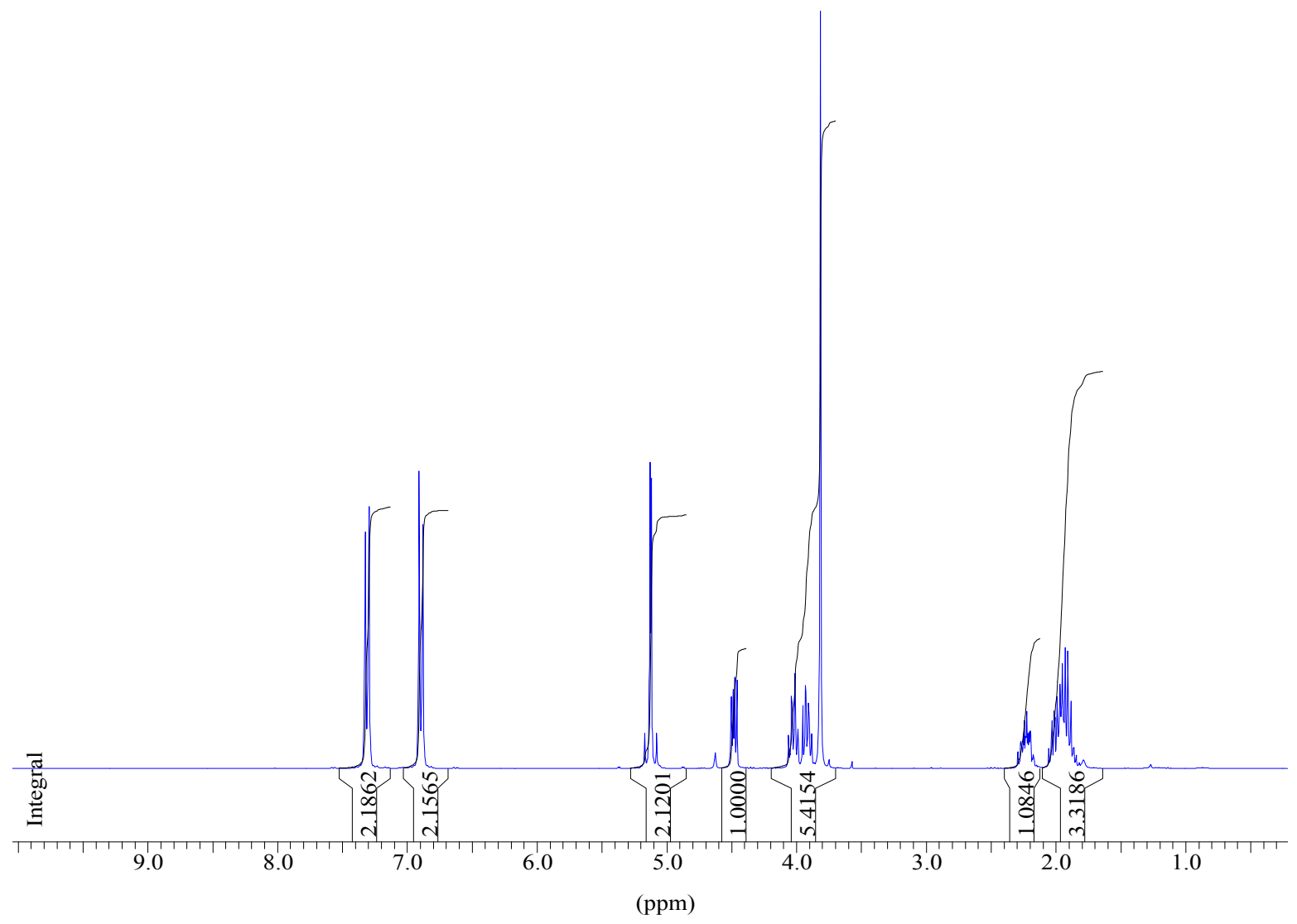




\section{Table 2 entry 11}<smiles>CO[R16](=O)N[C@@H](Cc1c[nH]c2ccccc12)C(=O)OC</smiles>

The spectral data obtained for this compound correspond to those described in the literature. ${ }^{1}$ HPLC retention time $3.81 \mathrm{~min}$.

Diastereomeric excess measured by HPLC using a Chiralpak AS, 250×4.6 mm. Chormatographic conditions: isocratic elution hexane: 2-propanol : diethylamine $85: 15: 0.1$, flow $1.0 \mathrm{~mL} / \mathrm{min}$. Retention times (min): 7.68 (minor), 10.44 (major).

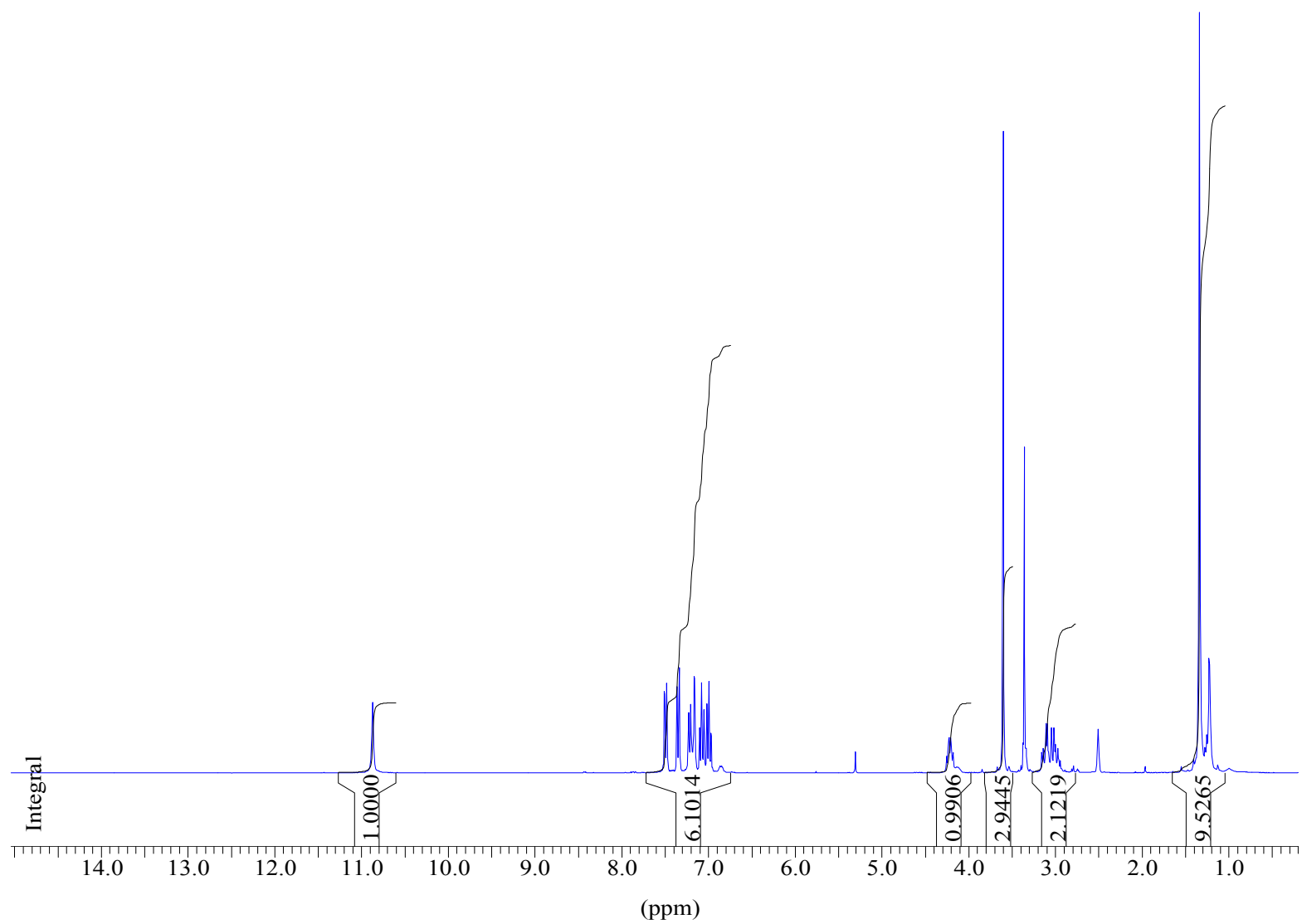

${ }^{1}$ Cardillo, G.; Gentilucci, L.; Tomasini, C.; Tomasoni, L. Tetrahedron: Asimm. 1995, 6, 1947-1955. 


\section{Table 2 entry 12}

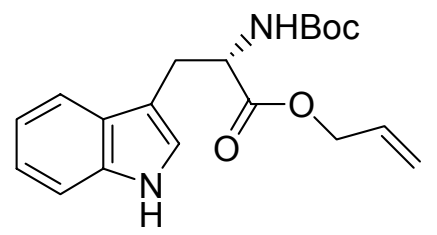

NMR data identical to what is reported in the literature. ${ }^{2}$

HPLC retention time $4.16 \mathrm{~min}$.

ESMS m/z 343.2 (M-H) ${ }^{-}$(neg. ion.)

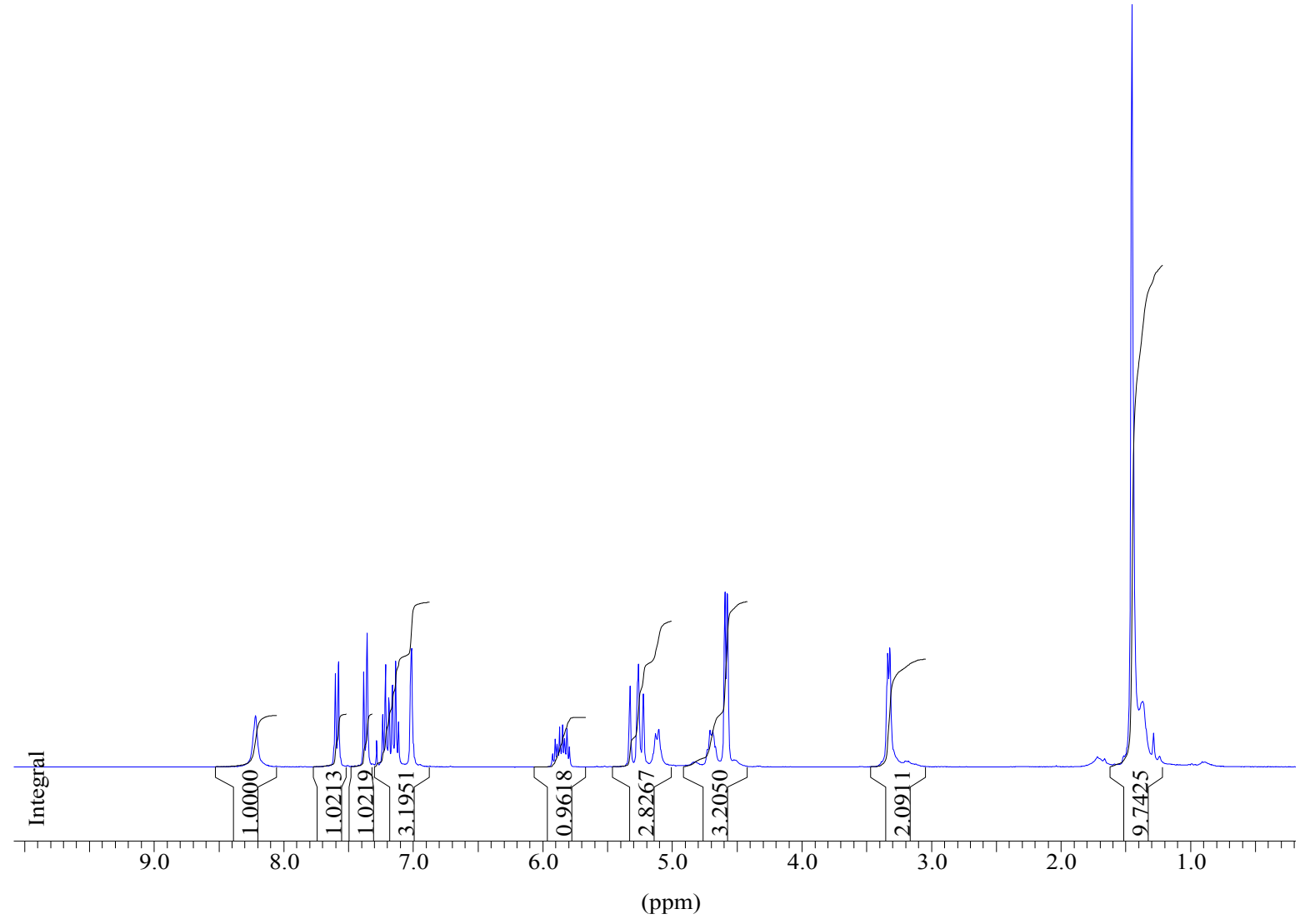




\section{Table 2 entry 13}

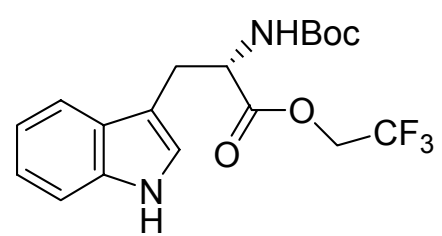

${ }^{1} \mathrm{H}$ NMR $\left(300 \mathrm{MHz} ; \mathrm{CDCl}_{3}\right) \delta_{\mathrm{H}}: 8.07(1 \mathrm{H}, \mathrm{bs}) ; 7.48(2 \mathrm{H}, \mathrm{d}, J=7.5 \mathrm{~Hz}) ; 7.29$ (2H, d, J= $\left.7.5 \mathrm{~Hz}\right)$; 7.19$7.02(2 \mathrm{H}, \mathrm{m}) ; 6.94(1 \mathrm{H}, \mathrm{d}, J=2.0 \mathrm{~Hz}) ; 4.95(1 \mathrm{H}, \mathrm{m}) ; 4.65(1 \mathrm{H}, \mathrm{m}) ; 4.50-4.22(2 \mathrm{H}, \mathrm{m}) ; 3.24(2 \mathrm{H}, \mathrm{m})$; $1.34(9 \mathrm{H}, \mathrm{s})$.

HPLC retention time $4.30 \mathrm{~min}$.

ESMS m/z $385.2(\mathrm{M}-\mathrm{H})^{-}$(neg. ion.)

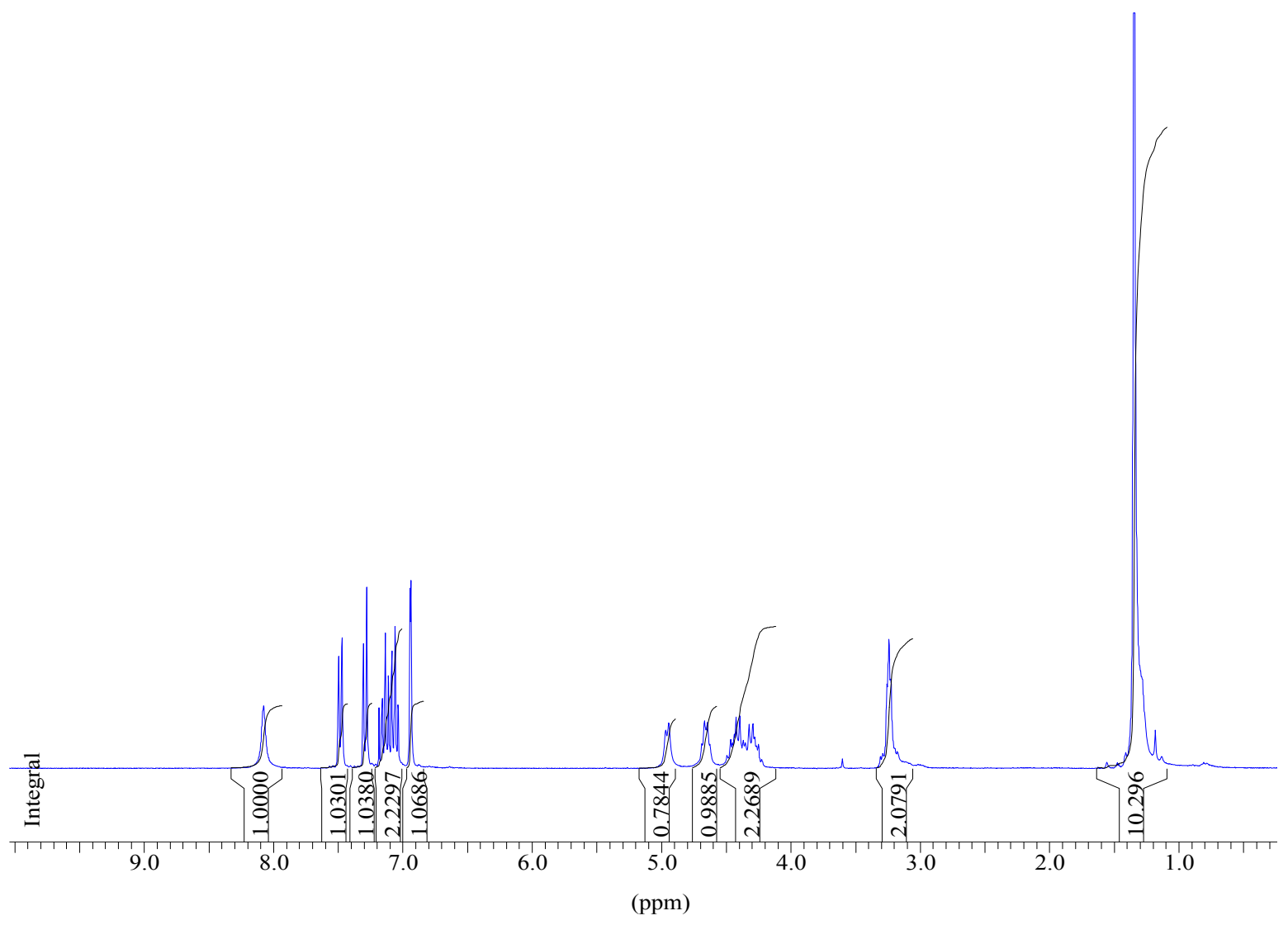




\section{Table 2 entry 14}

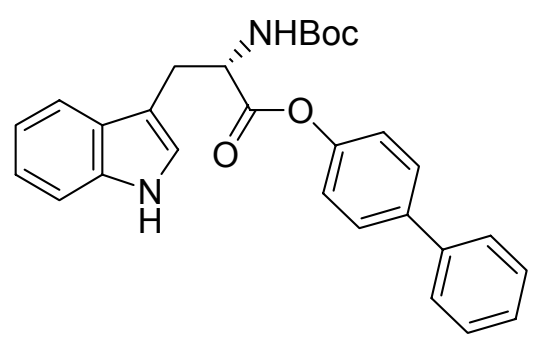

${ }^{1} \mathrm{H}$ NMR $\left(300 \mathrm{MHz} ; \mathrm{CDCl}_{3}\right) \delta_{\mathrm{H}}: 8.16(1 \mathrm{H}, \mathrm{bs}) ; 7.58(2 \mathrm{H}, \mathrm{d}, J=7.5 \mathrm{~Hz}) ; 7.48-7.23(9 \mathrm{H}, \mathrm{m}) ; 6.91-$ 6.87(2H, m); $5.12(1 \mathrm{H}, \mathrm{m}) ; 4.83(1 \mathrm{H}, \mathrm{m}) ; 3.41-3.35(2 \mathrm{H}, \mathrm{m}) ; 1.37(9 \mathrm{H}, \mathrm{s})$.

HPLC retention time $5.06 \mathrm{~min}$.

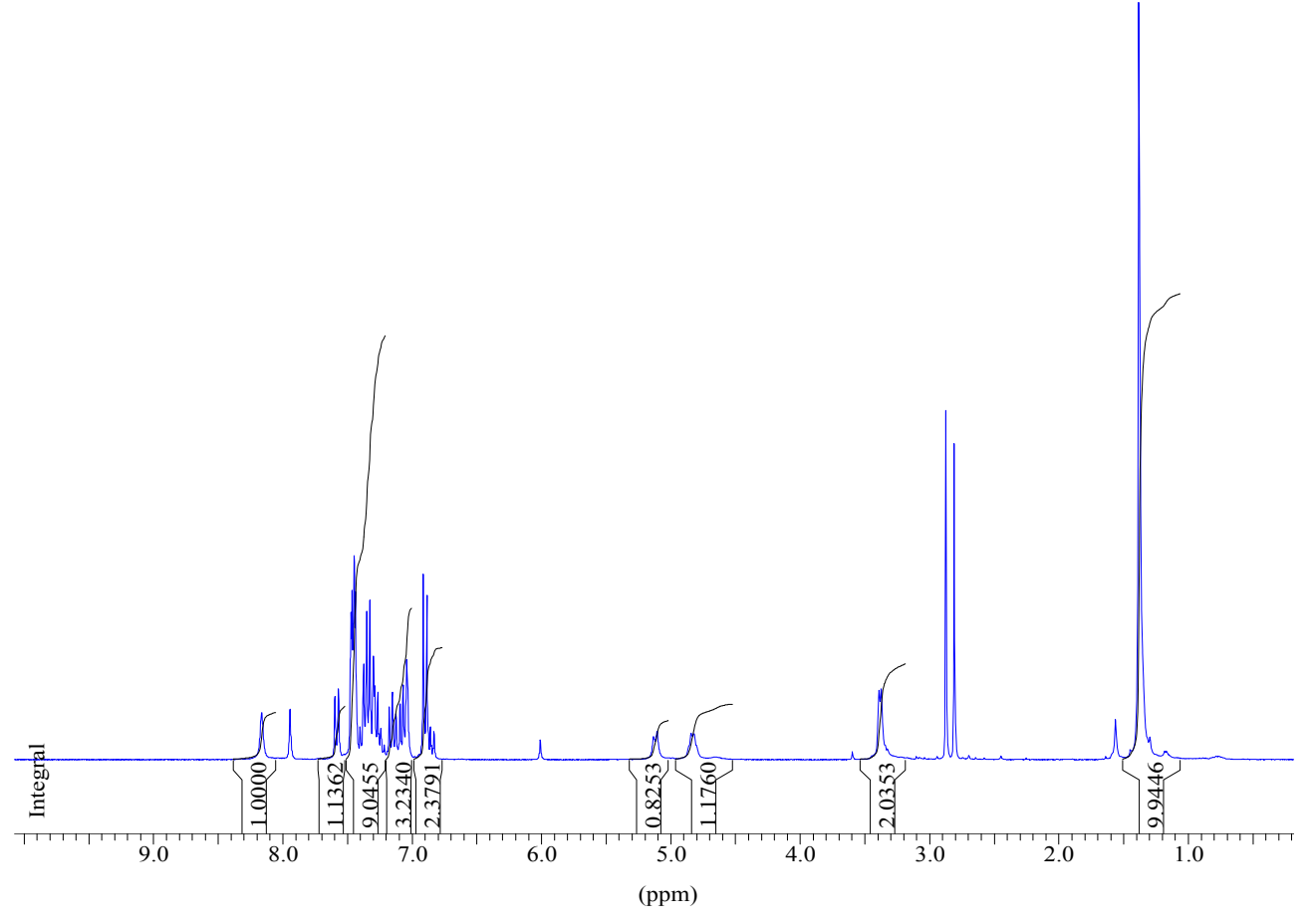




\section{Table 2 entry 15}

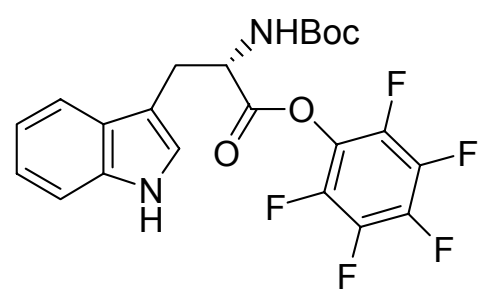

${ }^{1} \mathrm{H}$ NMR $\left(300 \mathrm{MHz} ; \mathrm{CDCl}_{3}\right) \delta_{\mathrm{H}}: 8.58(1 \mathrm{H}, \mathrm{bs}) ; 7.55(2 \mathrm{H}, \mathrm{d}, J=7.5 \mathrm{~Hz}) ; 7.29(2 \mathrm{H}, \mathrm{d}, J=7.5 \mathrm{~Hz}) ; 7.16-$ $7.03(2 \mathrm{H}, \mathrm{m}) ; 7.01(1 \mathrm{H}, \mathrm{s}) ; 5.06(1 \mathrm{H}, \mathrm{d}, J=7.5 \mathrm{~Hz}) ; 4.90(1 \mathrm{H}, \mathrm{m}) ; 3.42(1 \mathrm{H}, \mathrm{dd}, J=15.0 \mathrm{~Hz}, J=5.5 \mathrm{~Hz})$; $3.42(1 \mathrm{H}, \mathrm{dd}, J=15.0 \mathrm{~Hz}, J=6.0 \mathrm{~Hz}) ; 1.33(9 \mathrm{H}, \mathrm{s})$.

${ }^{19} \mathrm{~F}$ NMR $\left(\mathrm{CDCl}_{3}\right)$ : $-152.7 ;-157.9 ;-162.4 \mathrm{ppm}$.

HPLC retention time $4.90 \mathrm{~min}$.

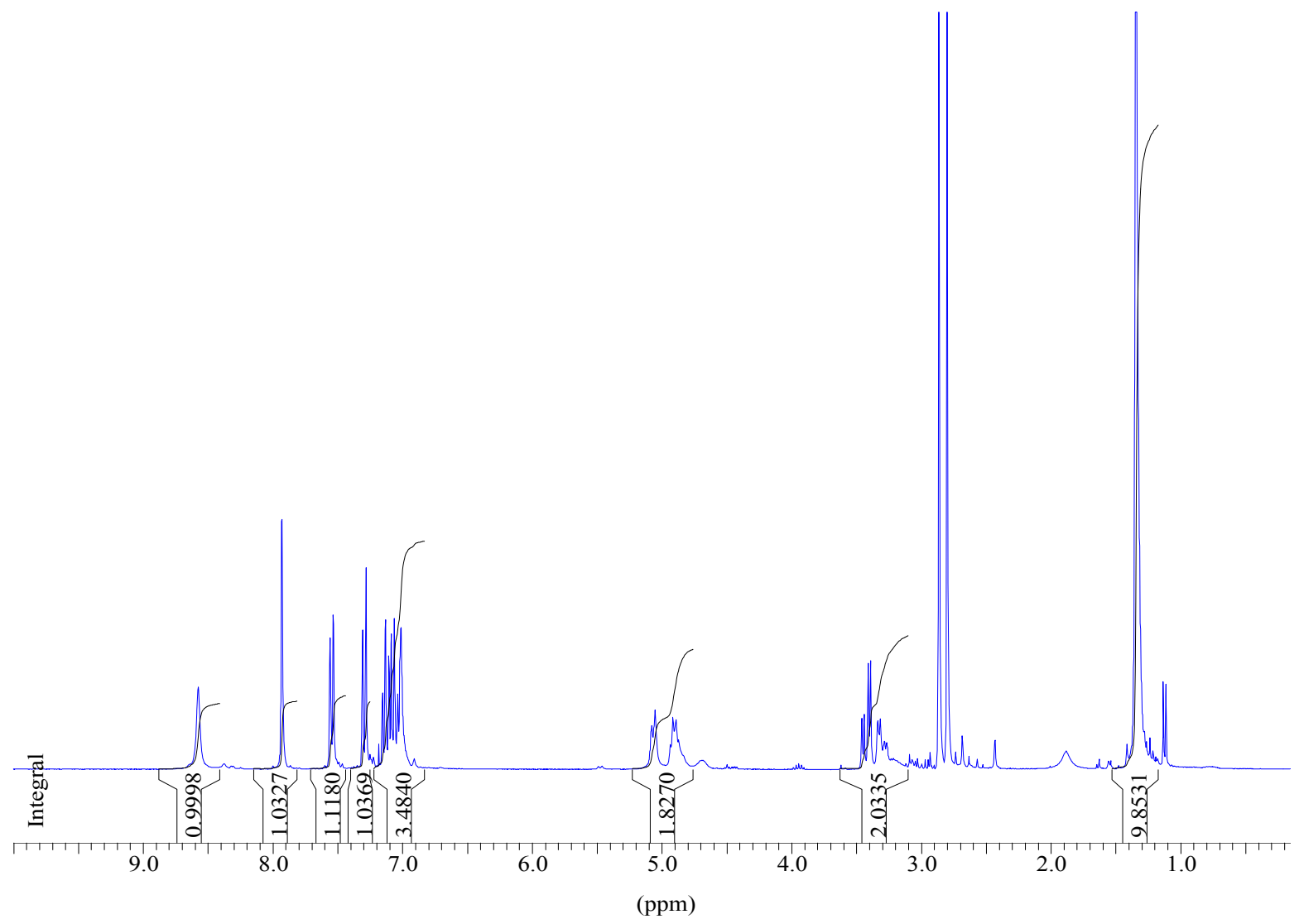

